\title{
THE EULER CHARACTERISTIC AS AN OBSTRUCTION TO COMPACT LIE GROUP ACTIONS
}

\author{
VOLKER HAUSCHILD
}

\begin{abstract}
Actions of compact Lie groups on spaces $X$ with $H^{*}(X, \mathbf{Q}) \cong$ $\mathrm{Q}\left[x_{1}, \ldots, x_{n}\right] / I_{0}, Q \in I_{0}$ a definite quadratic form, $\operatorname{deg} x_{i}=2$, are considered. It is shown that the existence of an effective action of a compact Lie group $G$ on such an $X$ implies $\chi(X) \equiv O(|W G|)$, where $\chi(X)$ is the Euler characteristic of $X$ and $|W G|$ means the order of the Weyl group of $G$. Moreover the diverse symmetry degrees of such spaces are estimated in terms of simple cohomological data. As an application it is shown that the symmetry degree $N_{t}(G / T)$ is equal to $\operatorname{dim} G$ if $G$ is a compact connected Lie group and $T \subset G$ its maximal torus. Effective actions of compact connected Lie groups $K$ on $G / T$ with $\operatorname{dim} K=\operatorname{dim} G$ are completely classified.
\end{abstract}

In the following let $G$ be a compact connected Lie group; we consider $G$-actions on spaces $X$ under the following general assumptions

(i) $X$ is a paracompact Hausdorff space of finite rational cohomology dimension or is compact,

(ii) $G$ acts on $X$ continuously with finitely many orbit types.

These are the usual assumptions made in the cohomology theory of compact transformation groups. If we use Alexander-Spanier-Čech cohomology with compact supports, these conditions guarantee the existence of a localization isomorphism, see $[2,12]$. The coefficient field $k$ of the cohomology is always $\mathbf{Q}$ or another field of characteristic 0 . We shall omit this in the notations and write simply $H^{*}(X ; k)=$ $H^{*}(X)$. In this paper we are mainly concerned with actions of $G$ on spaces $X$ which satisfy the following cohomological property

The cohomology ring $H^{*}(X)$ is generated as a Q-algebra by finitely many elements of degree two, i.e., $H^{*}(X) \cong P / I_{0}, P=$ $\mathbf{Q}\left[x_{1}, \ldots, x_{n}\right], \operatorname{deg} x_{i}=2$, and the defining ideal $I_{0}$ contains a definite quadratic form $Q$.

Here $Q$ is considered as a quadratic form on the vector space $\mathbf{Q}^{n}$. In the following such spaces will be called spaces of $q$-type. Examples are the homogeneous spaces $G / T, G$ a compact semisimple Lie group, $T \subset G$ a maximal torus. This follows from the classical result of Borel [1, Theorem 20.3];

$$
H^{*}(G / T) \cong H^{*}\left(B_{T}\right) / I_{0},
$$

Received by the editors September 30, 1985 .

1980 Mathematics Subject Classification (1985 Revision). Primary 57S10, 57S15, 57S25, 57R91, 55P62.

Key words and phrases. Topological transformation groups, cohomology theory of topological transformation groups, symmetry degree, homogeneous spaces, rational homotopy groups, spaces of $F$-type. 
where $I_{0}$ is the ideal generated by the invariants of the natural action of the Weyl group $W G$ on the augmentation ideal $H_{+}^{*}\left(B_{T}\right)$. If we denote by $L G$ the real Lie algebra of $G$, by $L T$, respectively, the real Lie algebra of $T$, then by the compactness of $G$ the Killing form $\kappa: L G \times L G \rightarrow \mathbf{R}$ gives rise to a (negatively) definite quadratic form on $L G$. The restriction of $\kappa$ to $L T$ gives a definite quadratic form $Q$ on $L T$ which is invariant under the Weyl group. Moreover, $Q$ is defined over $\mathbf{Q}$, i.e., has rational coefficients. So $Q$ gives a definite quadratic form in the ideal $I_{0}$ which will also be called $Q$. In this way we can consider spaces of $q$-type as generalizations of homogeneous spaces $G / T$. It comes out that spaces of $q$-type have a very nice behavior under compact Lie group actions. Before we begin with the enumeration of our results concerning actions on spaces of $q$-type, we introduce some notations and conventions. In [12] W. Y. Hsiang defines the topological symmetry degree $N_{t}(X)$ of a space $X$ as

$$
N_{t}(X)=\max \{\operatorname{dim} G ; G \text { compact Lie, } G \text { acts effectively on } X\} \text {. }
$$

Moreover, he defines the semisimple symmetry degree $N_{t}^{\text {ss }}(X)$ as $N_{t}^{\text {ss }}(X)=$ $\max \{\operatorname{dim} G ; G$ compact semisimple, $G$ acts effectively on $X\}$ and the torus symmetry degree $T_{t}(X)$ as

$$
T_{t}(X)=\max \{\text { rk } G ; G \text { a torus, } G \text { acts effectively on } X\} .
$$

Our main goal in this paper is to give some very sharp estimations for all these numbers in the case that $X$ is a space of $q$-type. Let $k$ denote a field. If $A$ is a local $k$-algebra, we denote by $\operatorname{edim} A$ the embedding dimension of $A$. By definition we have edim $A=\operatorname{dim}_{k} m_{A} / m_{A}^{2}$, where $m_{A}$ is the maximal ideal of $A$. In the case that $A$ is a positively graded local $k$-algebra, $m_{A}$ is the augmentation ideal $A_{+}$of $A$. The embedding dimension can also be interpreted as the minimal number of generators of $A$ as a $k$-algebra, i.e., if $n=\operatorname{edim} A$, then $n$ is the lowest integral number such that $A$ can be written as $A=k\left[x_{1}, \ldots, x_{n}\right] / I, I \subset\left(x_{1}, \ldots, x_{n}\right)$ a defining ideal. If $A$ is positively graded, the $x_{i}$ can be chosen as homogeneous generators such that $I$ is generated by homogeneous polynomials with respect to the grading on the polynomial ring. So, for example, edim $H^{*}(G / T)$ is equal to rk $G$.

THEOREM 1. Let $X$ be a closed connected compact oriented manifold of q-type. Then we have the inequality $T_{t}(X) \leqslant \operatorname{edim} H^{*}(X)$.

Thus it follows that the highest rank $r$ of a torus $S$ which acts effectively on a homogeneous space $G / T$ is equal to $r=\operatorname{rk} G$.

The existence of a definite quadratic form in the defining ideal of $H^{*}(X)$ can also be used to get more information on the cohomology of the fixed space $X^{G}$ of a torus action. Our first result in this direction is

THEOREM 2. Let $X$ be a space of $q$-type. If a torus $G$ acts on $X$ with fixed space $X^{G}$ such that $H^{*}\left(X, X^{G}\right) \neq 0$, then for every connected component $F$ of $X^{G}$ we have the inequality

$$
\operatorname{edim} H^{*}(F)<\operatorname{edim} H^{*}(X) \text {. }
$$

The application of Theorem 2 to a special case gives a new result in P. A. Smith theory. 
TheOrem 3. Let $X$ be a space of q-type with edim $H^{*}(X)=2$. If a torus $G$ acts on $X$ with fixed space $X^{G}$ such that $H^{*}\left(X, X^{G}\right) \neq 0$, then

$$
X^{G} \sim{ }_{\mathbf{Q}} S^{2}+\cdots+S^{2}+\text { isolated points. }
$$

Moreover, if $d$ is the number of 2-spheres in the above decomposition and $r$ denotes the number of isolated points, we have $\chi(X)=2 d+r$, where $\chi(X)$ is the Euler characteristic of $X$.

Here the symbol " " means rational homotopy equivalence. In the case $X \sim{ }_{\mathrm{Q}} K / T, K$ a compact Lie group with rk $K=2, T \subset K$ a maximal torus, we obtain the following list of possible fixed space cohomologies of torus actions on $X$.

\begin{tabular}{|c|c|c|c|}
\hline$\frac{\mathrm{SU}(2) \times \mathrm{SU}(2)}{T^{2}}$ & $\frac{\mathrm{SU}(3)}{T^{2}}$ & $\frac{\operatorname{Spin}(5)}{T^{2}}$ & $G_{2} / T$ \\
\hline $2 S^{2}$ & $3 S^{2}$ & $4 S^{2}$ & $6 S^{2}$ \\
$2 p+S^{2}$ & $2 p+2 S^{2}$ & $2 p+3 S^{2}$ & $2 p+5 S^{2}$ \\
$4 p$ & $4 p+S^{2}$ & $4 p+2 S^{2}$ & $4 p+4 S^{2}$ \\
$6 p$ & $6 p+S^{2}$ & $6 p+3 S^{2}$ \\
& & $8 p$ & $8 p+2 S^{2}$ \\
& & $10 p+S^{2}$ \\
& & \\
\cline { 2 - 2 } & &
\end{tabular}

It follows from a general existence principle in [9] that all these cases do indeed occur as fixed spaces of circle actions on finite $\mathrm{CW}$-complexes with the Q-cohomology of the above $K / T$. We mention that the fixed spaces in the first row of the table are realized by linear $S^{1}$-actions. The same is true for the fixed spaces consisting only of isolated points. The other possibilities occur only for nonlinear actions. It should be remarked that the second column has already been given in [11].

We now come to the results concerning actions of semisimple and arbitrary compact groups on spaces of $q$-type. An action of a compact Lie group $G$ on a space $X$ is called cohomologically effective (c-effective) if $H^{*}\left(X, X^{S}\right) \neq 0$ for every subtorus $S \subset G$ of positive dimension. If $X$ is a closed connected compact oriented manifold of $q$-type, then it is easy to see that an effective action is always c-effective. The following theorem is perhaps the most striking result of this paper and gave the title of the work.

THEOREM 4. Let $X$ be a space of $q$-type, and let $G$ be a semisimple compact connected Lie group which acts c-effectively on $X$. Then the Euler characteristic $\chi(X)$ of $X$ is divided by the order $|W G|$ of the Weyl group of $G$.

A short glance at the possible orders of Weyl groups teaches us that Theorem 4 gives strong restrictions for the possible semisimple Lie groups $G$ which can act on a given manifold of $q$-type. If, for example, $\chi(X)=2 m, m \not \equiv O(2)$ and $m \not \equiv O(3)$, then the only semisimple Lie groups which can act on $X$ are $\operatorname{SO}(3)$ and $\operatorname{Spin}(3)$. See 
Theorem 6.2 and also the example of a Spin(3)-action in \$6. A simple corollary of Theorem 4 is the following observation, which is difficult to find in the literature although it is surely known:

ObSERVATION. If $H$ is a closed connected semisimple subgroup of a compact semisimple Lie group $G$, then $|W G|=s \cdot|W H|$ for some positive integer $s$.

But we can show even more than Theorem 4 . Denote by $2 c_{1}-1,2 c_{2}-1, \ldots, 2 c_{r}$ -1 the degrees of the different rational homotopy groups of $G$. In rational homotopy theory a space $X$ is called an $F$-space if the pseudo-dual rational homotopy $\pi_{\psi}^{*}(X)$ and $H^{*}(X ; \mathbf{Q})$ are finite-dimensional vector spaces. If $X$ is 1 -connected this is equivalent to $\operatorname{dim}_{\mathbf{Q}} \pi_{*}(X) \otimes_{\mathbf{Z}} \mathbf{Q}<\infty$ and $\operatorname{dim}_{\mathbf{Q}} H^{*}(X ; \mathbf{Q})<\infty$. Denote by $2 b_{1}-1,2 b_{2}-1, \ldots, 2 b_{n}-1$ the degrees of the odd dimensional rational homotopy groups of $X$. It follows from a theorem of Halperin [6] that the cohomology ring of a 1-connected $F$-space which is also of $q$-type is always a complete intersection.

THEOREM 5. Let $X$ be a 1-connected q-space which is also an F-space. Assume that $X$ is a locally finite $C W$-complex. Let $G$ be a compact connected Lie group which acts c-effectively on $X$ in such a way that the space $X_{G}=E_{G} \times{ }_{G} X$ has the homotopy type of a $C W$-complex. Then the pair of sequences $B=\left(b_{1}, \ldots, b_{n}\right)$ and $A=\left(c_{1}, \ldots, c_{r}\right)$ satisfies the arithmetic condition (A.C.) of [5].

As a corollary of Theorem 5 we obtain the following divisibility condition for Poincaré polynomials.

THEOREM 6. Let $X$ be a 1-connected q-space which is also an F-space and assume that $X$ is a locally finite $C W$-complex. Let $G$ be a compact connected semisimple Lie group which acts c-effectively on $X$ in such a way that $X_{G}$ has the homotopy type of a $C W$-complex. If $T \subset G$ is a maximal torus, then the Poincaré-polynomial $P(G / T ; t)$ of $G / T$ divides the Poincare polynomial $P(X ; t)$ of $X$ such that the quotient $P(X ; t) / P(G / T ; t)$ is a polynomial with nonnegative integral coefficients.

It is clear that Theorem 4 can be recovered from Theorem 6 in this case. A further result is an upper bound for the semisimple symmetry degree:

THEOREM 7. Let $X$ be a space of q-type. If a compact connected semisimple Lie group $G$ acts c-effectively on $X$ then

$$
\operatorname{dim} G \leqslant \operatorname{cd}_{\mathbf{Q}} X+\operatorname{edim} H^{*}(X) .
$$

Here $\operatorname{cd}_{\mathbf{Q}} X$ is the $\mathbf{Q}$-cohomology dimension of $X$. Thus we obtain

THEOREM 8. If $X$ is a compact connected closed oriented manifold of q-type, then $N_{t}^{\mathrm{ss}}(X) \leqslant \operatorname{dim} X+\operatorname{edim} H^{*}(X)$.

Then we give the following application to homogeneous spaces

THEOREM 9. Let $G$ be a compact connected semisimple Lie group, $T \subset G$ a maximal torus. Let $X=G / T$. Then $N_{t}(X)=N_{t}^{\mathrm{ss}}(X)=\operatorname{dim} G$. Moreover, if a compact connected Lie group $K$ with $\operatorname{dim} K=\operatorname{dim} G$ acts effectively on $X$, then $K$ is locally isomorphic to $G$ and $K$ acts on $X$ by left translations up to conjugation. 
In the last section we give a much stronger estimation of $N_{t}^{\mathrm{ss}}(X)$ in terms of a number theoretical function $\Phi: \mathbf{N} \rightarrow \mathbf{N}$ which is given as follows:

$$
\Phi(n)=\max \{\operatorname{dim} G ; G \text { compact semisimple, }|W G| / n\} .
$$

THEOREM 10. If $X$ is a compact connected closed oriented manifold of q-type, then $N_{t}^{\mathrm{ss}}(X) \leqslant \Phi(\chi(X))$.

Obviously, this is almost a direct consequence of Theorem 4. The work is finished with a construction of Spin(3)-actions on finite CW-complexes of $q$-type showing that the upper bound $N_{t}^{\mathrm{ss}}(X) \leqslant 3$ for a closed compact connected oriented manifold $X$ of $q$-type with $\chi(X) \not \equiv O(4)$ and $\chi(X) \not \equiv O(6)$ is at least sharp in the category of finite $\mathrm{CW}$-complexes with cellular $G$-action.

1. Localization and other preliminary facts. The main goal of this section is to collect some more or less well-known facts from the cohomology theory of compact transformation groups which are necessary for the subsequent considerations. We begin with some commutative algebra. To fix notations, let $k$ be a field of char $k=0$. Denote by $k\left[x_{1}, \ldots, x_{n}\right]$ the graded commutative polynomial algebra in the indeterminants $x_{1}, \ldots, x_{n}$ of positive degree. Note $\operatorname{deg} x_{i} \equiv O(2), i=1, \ldots, n$. If $B$ is a positively graded local $k$-algebra, let $B_{+} \subset B$ denote the augmentation ideal.

TheOREM 1.1. Let $R=k\left[t_{1}, \ldots, t_{r}\right], P=k\left[x_{1}, \ldots, x_{n}\right]$, and $I_{0} \subset P_{+}$the ideal generated by the homogeneous elements $f_{1}, \ldots, f_{m}$. Set $A_{0}=P / I_{0}$ and assume $\operatorname{dim}_{k} A_{0}<\infty$. If $A$ is a homogeneous $R$-algebra, free as an $R$-module, such that $A \otimes_{R} k=A_{0}$ in the graded sense, then $A=P_{R} / I$, where $P_{R}=R \otimes_{k} P$ and $I \subset P_{R}$ is an ideal generated by homogeneous elements of the form $1 \otimes f_{j}-r_{j}, j=1, \ldots, m$, where the $r_{j}$ are elements of $R_{+} P_{R}$. In particular, $A$ is a finitely generated $R$-module of $\mathrm{rk}_{R} A=\operatorname{dim}_{k} A_{0}$.

Proof. From $A \otimes_{R} k=A_{0}$ it follows that $\operatorname{rk}_{R} A=\operatorname{dim}_{k} A_{0}$, i.e., $A$ is finitely generated.

Let $p: A \rightarrow A_{0}$ denote the canonical projection, let $\bar{X}_{1}, \ldots, \bar{X}_{n} \in A$ be homogeneous elements with $p\left(\bar{X}_{i}\right)=\bar{x}_{i}$, where $\bar{x}_{i}$ is the remainder of $x_{i} \bmod I_{0}$. Then $A_{0}$ is generated as a $k$-vector space by 1 and some polynomials $p_{j}\left(\bar{x}_{1}, \ldots, \bar{x}_{n}\right), j=$ $1, \ldots, N-1, N=\operatorname{dim}_{k} A_{0}$.

Thus it follows from the lemma of Nakayama that $A$ as a $R$-module is generated by 1 and the polynomials $p_{j}\left(\bar{X}_{1}, \ldots, \bar{X}_{n}\right), j=1, \ldots, N-1$. We write $P_{R}=$ $R\left[X_{1}, \ldots, X_{n}\right], X_{i}=1 \otimes x_{i}$. Then the homomorphism $P_{R} \rightarrow A, X_{i} \rightarrow \bar{x}_{i}$ is surjective, i.e., we can write $A=P_{R} / I$, where $I$ is a certain ideal. Let $F_{j}=1 \otimes f_{j} \in P_{R}$ and denote by $\bar{F}_{j}$ its image in $A$, then $p\left(\bar{F}_{j}\right)=0 \in A_{0}$. This shows $\bar{F}_{j} \in R_{+} A$. Let $r_{j} \in R_{+} P_{R}, j=1, \ldots, m$, be homogeneous elements such that $\bar{F}_{j}-\bar{r}_{j}=0$ in $A$. Then $F_{j}-r_{j} \in I, j=1, \ldots, m$. Let $I^{\prime} \subset I$ be the ideal generated by the $F_{j}-r_{j}$, let $\varphi: P_{R} / I^{\prime} \rightarrow P_{R} / I$ denote the obvious quotient map, and let $K=\operatorname{ker} \varphi$. Then, by the freeness of $A$, the exact sequence $0 \rightarrow K \rightarrow P_{R} / I^{\prime} \rightarrow P_{R} / I \rightarrow 0$ induces an exact sequence of $k$-vector spaces after tensoring with $k$ over $R$. In particular, the induced homomorphism $\bar{\varphi}:\left(P_{R} / I^{\prime}\right) \otimes_{R} k \rightarrow A_{0}$ becomes an isomorphism. In other words, we 
have $\bar{K}=K \otimes_{R} k=0$. Because $K$ is a graded module over a positive graded polynomial ring, this implies $K=0$. So, $\varphi$ is an isomorphism, i.e., $I=I^{\prime}$.

Now let $X$ be a topological space, and let $G$ be a compact Lie group which acts on $X$. We then consider the fibration $X \rightarrow E_{G} \times{ }_{G} X \rightarrow B_{G}$ introduced by Borel. We put $X_{G}=E_{G} \times{ }_{G} X$ and $H_{G}^{*}(X)=H^{*}\left(X_{G}\right)$. Here we use Alexander-Spanier-Čech cohomology with compact supports. The coefficient field $k$ shall be omitted in the notations. We denote the projection in the above fiber bundle by $\pi$. If $R_{G}=H^{*}\left(B_{G}\right)$, the ring $H_{G}^{*}(X)$ is a $R_{G}$-algebra via $\pi^{*}$ and the cup product. Obviously, the above construction is functorial with respect to equivariant maps. The functor obtained in this way is called the ordinary equivariant cohomology with coefficients in $k$.

Let $X$ be a space such that $H^{*}(X)$ is generated by finitely many elements of even degree, i.e., $H^{*}(X)=k\left[x_{1}, \ldots, x_{n}\right] / I_{0}, \operatorname{deg} x_{i}=O(2), I_{0}=\left(f_{1}, \ldots, f_{m}\right)$, where the $f_{j}$ are homogeneous polynomials such that $\operatorname{dim}_{k} H^{*}(X)<\infty$. If $G$ acts on $X$, the Serre spectral sequence associated with the Borel fibering degenerates at the 2-term. This implies $H_{G}^{*}(X)$ is a free $R_{G}$-module. Let $R_{+}=R_{G_{+}+} \subset R_{G}$. The homomorphism $i^{*}: H_{G}^{*}(X) \rightarrow H^{*}(X)$ induced by the inclusion of the fiber $X \rightarrow X_{G}$ is then given by the projection map

$$
H_{G}^{*}(X) \rightarrow H_{G}^{*}(X) / R_{+} H_{G}^{*}(X) \cong H^{*}(X) .
$$

(See e.g. [1, Theorem 14.2].) Naturally, we have $H_{G}^{*}(X) / R_{+} H_{G}^{*}(X) \cong H_{G}^{*}(X) \otimes_{R_{G}} k$. Therefore, by Theorem 1.1, we get

$$
H_{G}^{*}(X)=R_{G}\left[X_{1}, \ldots, X_{n}\right] / I,
$$

where the defining ideal $I$ is generated by elements of the kind $F_{j}-r_{j}, r_{j} \in R_{+} P_{G}$, $F_{j}=1 \otimes f_{j}, j=1, \ldots, m$, where we have written $P_{G}=R_{G}\left[X_{1}, \ldots, X_{n}\right]$. The polynomials $r_{j}$ depend on the geometric behavior of the $G$-action. Thus we have to investigate what kind of polynomials $r_{j}$ can actually occur from a $G$-action. A partial answer to this question is furnished by the localization theorem in ordinary equivariant cohomology theory. Let $S \subset R_{G}$ be a multiplicatively closed subset and denote by $X^{S} \subset X$ the closed $G$-invariant subspace defined as follows: For every $y \in X$ we define an ideal $I_{x}$ in $R_{G}$ as the kernel $I_{x}=\operatorname{ker} \rho_{x}^{*}: R_{G} \rightarrow R_{G_{\mathrm{x}}}$ of the natural restriction map induced by the inclusion $G_{x} \subset G$ of the isotropy subgroup. Then we put

$$
X^{S}=\left\{y \in X ; I_{y} \cap S=\varnothing\right\}
$$

THEOREM 1.2 (LOCALIZATION THEOREM [12]). Let $X$ be a compact or paracompact Hausdorff space of finite $k$-cohomology dimension. Let the compact Lie group $G$ act on $X$ with finitely many orbit types. If $S \subset R_{G}$ is a multiplicatively closed subset, then the inclusion $X^{S} \subset X$ induces an isomorphism of graded $S^{-1} R_{g}$-algebras $S^{-1} H_{G}^{*}(X) \cong$ $S^{-1} H_{G}^{*}\left(X^{S}\right)$.

As an example, we consider the special case $S=R_{G}-\{0\}$. Then $X^{S}$ is the subset of all points $y \in X$ with $I_{y}=0$, i.e., where $\rho_{y}^{*}$ is injective. But $\rho_{y}^{*}: R_{G} \rightarrow R_{G_{r}}$ is injective iff $G_{y} \subset G$ contains a maximal torus $T_{y}$ of $G$. So we have $X^{S}=X^{(T)}$ where 
$X^{(T)} \subset X$ is the subset $X^{(T)}=\left\{y \in X\right.$; rk $\left.G_{y}=\operatorname{rk} G\right\}$. Obviously, $X^{(T)}=$ $\bigcup_{g \in G} X^{g T g^{-1}}$, where $T$ is a fixed maximal torus of $G$. So, we obtain

$$
S^{-1} H_{G}^{*}(X) \cong S^{-1} H_{G}^{*}\left(X^{(T)}\right) \text {. }
$$

In the case that $G$ is a torus, the set $X^{(T)}$ is equal to the fixed space $X^{G}$. We then obtain

$$
S^{-1} H_{G}^{*}(X) \cong S^{-1} R_{G} \otimes_{k} H^{*}\left(X^{G}\right),
$$

as follows from the fact that $H_{G}^{*}\left(X^{G}\right) \cong R_{G} \otimes_{k} H^{*}\left(X^{G}\right)$.

Theorem 1.3 (SPeCial CASE OF TheORem IV.1 in [12]). Let $X$ be a space obeying the hypotheses of the localization theorem. Furthermore assume $H^{*}(X)$ to be generated as a graded $k$-algebra by finitely many elements of even degree. Let $P_{G}=$ $R_{G}\left[X_{1}, \ldots, X_{n}\right]$ and $H_{G}^{*}(X)=P_{G} /$ I. If $S=R_{G}-\{0\}$, we write $J=S^{-1} I$ as an ideal in $S^{-1} P_{G}$.

(i) If $J=\bigcap_{i \in \Delta} J_{i}$ is the reduced primary decomposition of $J$, then there is a natural bijection between the set $\left\{J_{i}\right\}_{i \in \Delta}$ and the set $\pi_{0} X^{G}$ of connected components of the fixed set such that for the component $F_{i}$ we have

$$
S^{-1} R_{G} \otimes_{k} H^{*}\left(F_{i}\right) \cong S^{-1} P_{G} / J_{i} .
$$

Moreover, the localization isomorphism is given by the canonical map

$$
S^{-1} P_{G} / J \rightarrow \prod_{i \in \Delta} S^{-1} P_{G} / J_{i} .
$$

(ii) The set Ass $J$ of the associated prime ideals of $J$ is given by a family $\left\{\mathfrak{m}_{i}\right\}_{i \in \Delta}$ of maximal ideals $\mathrm{m}_{i}=\left(X_{1}-\omega_{i 1}, \ldots, X_{n}-\omega_{i n}\right)$, where the $\omega_{i j}$ are homogeneous elements of $R_{G} \subset S^{-1} R_{G}, \operatorname{deg} \omega_{i j}=\operatorname{deg} X_{j}$.

As a simple corollary we have

Lemma 1.1. Under the above assumptions the set Ass $I=\operatorname{Ass}_{P_{G}} P_{G} / I$ of the associated prime ideals is in a natural way isomorphic to the set of connected components $\pi_{0} X^{G}$ of the fixed set. Moreover, any $P \in$ Ass $I, I$ the defining ideal of $H_{G}^{*}(X)$, is of the form $P=\left(X_{1}-\omega_{1}, \ldots, X_{n}-\omega_{n}\right), \omega_{i} \in R_{G}$, $\operatorname{deg} \omega_{i}=\operatorname{deg} X_{i}$.

Proof. The first statement follows from (i) of Theorem 1.3. Because $H_{G}^{*}(X)$ is a free $R_{G}$-module, there is a bijection between the set Ass $_{P_{G}} P_{G} / I$ and the set

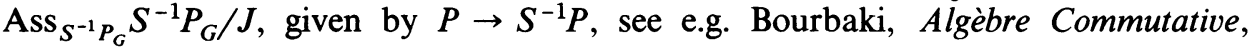
Chapter IV, 1.2.

We remark that in the case considered in Theorēm 1.3 it is not necessary to invert the full set $S=R_{G}-\{0\}$ for obtaining an isomorphism of localized rings $S^{-1} H_{G}^{*}(X) \cong S^{-1} H_{G}^{*}\left(X^{G}\right)$. This is a consequence of the fact that the $R_{G}$-module $H_{G}^{*}\left(X, X^{G}\right)$ has a finite set $Q_{j} \subset R_{G}$ of associated prime ideals which are all of the form $Q_{j}=\left(\omega_{j}\right), \omega_{j} \in H^{2}\left(B_{G}\right)$, i.e., are principal ideals. See, for example, [12, Theorem IV.6.]. Then it suffices to invert the element $\chi_{G}=\prod_{j} \omega_{j}$ thus getting the isomorphism

$$
H_{G}^{*}(X)\left[\frac{1}{\chi_{G}}\right] \cong H_{G}^{*}\left(X^{G}\right)\left[\frac{1}{\chi_{G}}\right]
$$


The element $\chi_{G}$ is then an equivariant Euler class in the sense of tom Dieck [2]. In the case $G=S^{1}$ the equivariant Euler class $\chi_{G}$ is proportional to the first Chern class of the universal principal bundle $S^{1} \rightarrow E_{S} \rightarrow B_{S}$ and can then be taken as the multiplicative generator of $H^{*}\left(B_{G}\right)=k[t]$. It is a simple observation from commutative algebra that a finitely generated $R_{G}$-module $M$ with $S^{-1} M=0$ vanishes already after localizing in a finitely generated multiplicative set.

Lemma 1.2. Let $X$ be a space satisfying the hypotheses of the localization theorem. Let $G=S^{1}$ and suppose $G$ acts on $X$ with finitely many orbit types. Let $K_{\eta}=$ $k[t] /(t-1)$ be the residue field of $\operatorname{Spec} R_{G}$ in the point $t=1$. Then the inclusion $X^{G} \subset X$ induces a not necessarily graded isomorphism of $K_{\eta}$-algebras

$$
H_{G}^{*}(X) \otimes_{R_{G}} K_{\eta} \cong H^{*}\left(X^{G} ; K_{\eta}\right) .
$$

This lemma has also been proved by V. Puppe in [15] where it is shown that under certain hypotheses the cohomology $H^{*}(F)$ of any fixed space component $F$ is a $k$-algebra generated by at most $n$ elements if $H^{*}(X)$ is generated by $n$ elements. In our language one could say that edim $H^{*}(F) \leqslant \operatorname{edim} H^{*}(X)$.

Proof. After the localization theorem the finitely generated $R_{G}$-module $H_{G}^{*}\left(X, X^{G}\right)$ is a torsion module. Because it is graded, the only annihilating prime ideal of $H_{G}^{*}\left(X, X^{G}\right)$ is given by $Q=(t)$. So it follows that $H_{G}^{*}(X)[1 / t]=$ $H_{G}^{*}\left(X^{G}\right)[1 / t]$. Tensoring this isomorphism with $k\left[t, t^{-1}\right] /(t-1)=K_{\eta}$ gives the desired result. (Use the universal coefficient theorem!)

2. A special case. As a very important special case we consider spaces $X$ whose $k$-cohomology $H^{*}(X)$ is generated by finitely many elements $x_{1}, \ldots, x_{n}$ of degree $\operatorname{deg} x_{i}=2$. Then again the ring $H_{G}^{*}(X)$ is a free finitely generated $R_{G}$-module. The long exact sequence of the couple $\left(X, X^{G}\right)_{G}$ degenerates into the short exact sequence

$$
0 \rightarrow H_{G}^{*}(X) \rightarrow H_{G}^{*}\left(X^{G}\right) \rightarrow H_{G}^{*}\left(X, X^{G}\right) \rightarrow 0,
$$

in particular, $H_{G}^{*}\left(X, X^{G}\right)$ is zero in even dimensions. This follows from the localization theorem because the image of a finitely generated torsion module in a finitely generated free module must be zero.

THEOREM 2.1. Let $X$ be a space satisfying the hypotheses of the localization theorem and assume $H^{*}(X)=k\left[x_{1}, \ldots, x_{n}\right] / I_{0}, \operatorname{deg} x_{i}=2, i=1, \ldots, n$. Let a torus $G$ act on $X$ with finitely many orbit types, put $H_{G}^{*}(X)=P_{G} / I, P_{G}=R_{G}\left[X_{1}, \ldots, X_{n}\right]$, then we have

(i) The set Ass I of associated prime ideals of the ideal I is in a natural way isomorphic to the set $\pi_{0} X^{G}$ of connected components of $X^{G}$. If $F_{j}$ is a connected component of $X^{G}$ and $s \in F_{j}$, then let the restriction homomorphism $H_{G}^{*}(X) \rightarrow$ $H_{G}^{*}(\{s\}) \cong R_{G}$ be given by $X_{i} \rightarrow \omega_{i j}, \omega_{i j} \in R_{G}$. Then the prime ideal of I corresponding to the component $F_{j}$ is given by $P_{j}=\left(X_{1}-\omega_{1 j}, \ldots, X_{n}-\omega_{n j}\right)$. 
(ii) If $I=\bigcap_{j \in \Delta} I_{j}$ is the reduced primary decomposition, then the sequence $(*)$ is given by the canonical exact sequence

$$
0 \rightarrow P_{G} / I \rightarrow \prod_{j \in \Delta} P_{G} / I_{j} \rightarrow \text { coker } \rightarrow 0 .
$$

Proof. The first statement is an immediate consequence of Theorem IV.1 in [12] and Lemma 1.1. For the second we observe that the homomorphism $r: H_{G}^{*}(X) \rightarrow$ $H_{G}^{*}(F)$, induced by the inclusion of a fixed space component $F \subset X$ is given by $X_{i} \rightarrow Y_{i}+\omega_{i}$ with $Y_{i} \in H^{2}(F), \omega_{i} \in H^{2}\left(B_{G}\right)$. It follows from the localization isomorphism that the localized homomorphism $S^{-1} r: S^{-1} H_{G}^{*}(X) \rightarrow S^{-1} H_{G}^{*}(F)$ is an epimorphism. The isomorphism $S^{-1} H_{G}^{*}(F) \cong S^{-1} R_{G} \otimes_{k} H^{*}(F)$ implies that $Y_{1}, \ldots, Y_{n} \in H^{2}(F)$ is a set of generators of the $k$-algebra $H^{*}(F)$. This shows $\bar{r}=r \otimes 1: H_{G}^{*}(X) \otimes_{R_{G}} k \rightarrow H_{G}^{*}(F) \otimes_{R_{G}} k \cong H^{*}(F)$ to be an epimorphism. If $C$ is the cokernel of $r$, then $\bar{C}=C \otimes_{R_{G}} k=0$. Because $C$ is finitely generated, the $R_{G}$-module $C$ must vanish by the lemma of Nakayama (or use the fact that a finitely generated graded module $C$ over a positive graded polynomial algebra $R$ is zero iff $\bar{C}=C \otimes_{R} k$ is zero). Thus it follows that $r$ is an epimorphism. Let $i_{j}: F_{j} \times B_{G} \subset X_{G}$ be the inclusion induced by $F_{j} \subset X$. It follows that $i_{j}^{*}: H_{G}^{*}(X) \rightarrow H_{G}^{*}\left(F_{j}\right)$ is an epimorphism for all fixed space components $F_{j}, j \in \Delta$. In other terms, $i_{j}^{*}$ is a projection map $i_{j}^{*}: P_{G} / I \rightarrow P_{G} / I_{j}$ for an ideal $I_{j} \subset P_{G}$ with $I \subset I_{j}$. Let $\left\{I_{j}\right\}_{j \in \Delta}$ be the family of ideals obtained in this way. We define the ideal $I^{\prime}$ as the intersection of all $I_{j}$. It follows that $I \subset I^{\prime}$. We have a commutative diagram

$$
\begin{aligned}
& H_{G}^{*}(X) \stackrel{i^{*}}{\rightarrow} \prod_{j \in \Delta} H_{G}^{*}\left(F_{j}\right) \\
& p \downarrow \quad \downarrow \cong \\
& P_{G} / I^{\prime} \quad \underset{\mu}{\rightarrow} \prod_{j \in \Delta} P_{G} / I_{j}
\end{aligned}
$$

Here the first horizontal arrow is induced by the inclusion of the fixed set, whereas $\mu$ is the obvious canonical map. The map $p$ is the projection induced by $I \subset I^{\prime}$. By the injectivity of $i^{*}$ it follows that $I^{\prime} \subset I$, i.e., $I=I^{\prime}$. So, $p$ is an isomorphism and we are done.

As a corollary we obtain

Lemma 2.1. Let $X$ be a space as in Theorem 2.1. Let a torus $G$ act on $X$ in such a way that $H^{*}\left(X, X^{G}\right) \neq 0$. Then $X^{G}$ consists of at least two components; in particular, there exists a connected component $F$ of $X^{G}$, such that the restriction homomorphism $H_{G}^{*}(X) \rightarrow H_{G}^{*}(\{q\}) \cong R_{G}, q \in F$, is nontrivial; i.e., the prime ideal $P \in$ Ass $I$ of the component $F$ is given by $P=\left(X_{1}-\omega_{1}, \ldots, X_{n}-\omega_{n}\right), \omega_{i} \neq 0$ for at least one index $i$, $1 \leqslant i \leqslant n$.

Proof. If there is one single component $F=X^{G}$, then $I$ is a primary ideal, so, by Theorem 2.1 we have $H_{G}^{*}(X)=P_{G} / I \cong H_{G}^{*}(F)$. But then it follows that $H^{*}(X) \cong$ $H^{*}(F)$, the isomorphism induced by the inclusion. So it follows that $H^{*}\left(X, X^{G}\right)=0$, a contradiction. Naturally, then two different prime ideals of $I$ must be different, so at least one of them is not of the form $P=\left(X_{1}, \ldots, X_{n}\right)$. 
Next, we consider the behavior of the associated prime ideals of the $P_{G}$-module $H_{G}^{*}(X)$ with respect to inclusions of subtori $K \subset G$. Here and in the sequel we need the following theorem

THEOREM 2.2. Let $G$ be a compact Lie group, $X$ a G-space, $K \subset G$ a subgroup. Let $\rho: R_{G} \rightarrow R_{K}$ be the restriction map induced by the inclusion. If either $R_{K}$ is a free module over $R_{G}$ or $H_{G}^{*}(X)$ is a free module over $R_{G}$, then the equivariant cohomology $H_{K}^{*}(X)$ of the induced $K$-action is given by $H_{K}^{*}(X) \cong H_{G}^{*}(X) \otimes_{R_{G}} R_{K}$.

Proof. We consider the following fiber square:

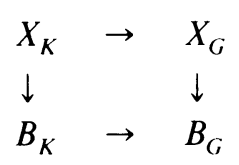

The corresponding Eilenberg-Moore spectral sequence has $E_{2}$-term equal to $E p^{q}=$ $\operatorname{Tor}_{R_{i}}^{p q}\left(H_{G}^{*}(X), R_{K}\right)$. By hypothesis $E q^{q}=0$ for $q \neq 0$ and

$$
E_{2}^{* .0}=\operatorname{Tor}_{R_{i}}^{* .0}\left(H_{G}^{*}(X), R_{K}\right) \cong H_{G}^{*}(X) \otimes_{R_{G}} R_{K} .
$$

So, the $E_{2}$-term consists of one line and the spectral sequence degenerates at the 2-term. Because of $E_{n} \Rightarrow H^{*}\left(X_{K}\right)=H_{K}^{*}(X)$ the result follows.

Let $\tilde{\rho}: P_{G} \rightarrow P_{K}$ be the homomorphism induced by $\rho: R_{G} \rightarrow R_{K}$. Then from the above theorem, we get

Lemma 2.3. Let $H_{G}^{*}(X)$ be a free $R_{G}$-module, $H_{G}^{*}(X)=P_{G} / I$, let the defining ideal I be generated by the elements $F_{1}, \ldots, F_{m} \in P_{G}$. Then the equivariant cohomology $H_{K}^{*}(X)$ is given by $H_{K}^{*}(X)=P_{K} / J$, where $J$ is generated by the elements $\tilde{\rho}\left(F_{1}\right), \ldots, \tilde{\rho}\left(F_{m}\right)$.

The proof is clear and shall be omitted.

If a $\subset P_{G}$ is an ideal write $a_{1 K}$ for the ideal generated in $P_{K}$ by the elements $\tilde{\rho}(F), F \in \mathfrak{a}$. If, for example, $Q \subset P_{G}$ is an ideal of the form $Q=\left(X_{1}-\omega_{1}, \ldots, X_{n}\right.$ $\left.-\omega_{n}\right), \omega_{i} \in R_{G}$, then the ideal $Q_{\mid K}$ in $P_{K}$ is given by $Q_{\mid K}=\left(X_{1}-\rho\left(\omega_{1}\right), \ldots, X_{n}\right.$ $\left.-\rho\left(\omega_{n}\right)\right)$.

We consider the following algebraic situation: Let $R$ be a local $k$-algebra, $R / m_{R}=k$, and let $S$ be a local $k$-algebra $S / m_{S}=k$, assume $S$ to be an integral domain. Let $P_{R}=R\left[X_{1}, \ldots, X_{n}\right]$ be the polynomial algebra in $n$ indeterminants. Let $\rho: R \rightarrow S$ be a surjective homomorphism of local rings. Let $P_{S}=S\left[X_{1}, \ldots, X_{n}\right]$ and let $\tilde{\rho}: P_{R} \rightarrow P_{S}$ be the surjective homomorphism induced by $\rho$. If $a \subset P_{R}$ is an ideal, denote by $a_{S} \subset P_{S}$ the ideal generated by all elements $\tilde{\rho}(F), F \in \mathfrak{a}$. We then have

Lemma 2.4. Let the situation be as above, assume $I \subset P_{R}$ to be an ideal such that $A=P_{R} / I$ is a free $R$-module, let $B=P_{S} / I_{S}=A \otimes_{R} S$. Then $B$ is a free $S$-module and the associated prime ideals of $I_{S}$ are given by Ass $I_{S}=\left\{Q_{S} ; Q \in\right.$ Ass $\left.I\right\}$.

Proof. Because $A$ is a free $R$-module, it is Cohen-Macaulay. So, the ideal $I$ is unmixed, i.e., cannot have embedded components. Thus the associated prime ideals of $I$ are completely determined by the variety $V(I)$ of $I$ in $\operatorname{Spec} P_{R}$. It is clear that $B$ 
is a free module over $S$; moreover, we have $V\left(I_{S}\right)=V(I) \cap \operatorname{Spec} P_{S}$. By the same arguments the prime ideals of $I_{S}$ are determined by $V\left(I_{S}\right)$. If $W^{\prime}$ is an irreducible component of $V\left(I_{S}\right)$, then there exists an irreducible component $V^{\prime}$ of $V(I)$ such that $W^{\prime}=V^{\prime} \cap \operatorname{Spec} P_{S}$. So, the prime ideal $p^{\prime}$ of $W^{\prime}$ is of the form $\mathfrak{p}^{\prime}=\mathfrak{p}_{S}$ for a prime ideal $p \in$ Ass $I$.

As a corollary we obtain

LemMA 2.5. Let $G$ be a torus which acts on a space $X$ such that $H_{G}^{*}(X)=P_{G} / I$ is a free $R_{G}$-module. Let $K \subset G$ be a subtorus and write $H_{K}^{*}(X)=P_{K} / J$ as in Lemma 2.3. Then Ass $J=\left\{\left.Q\right|_{K} ; Q \in\right.$ Ass $\left.I\right\}$.

Geometrically, this can be interpreted as follows.

Lemma 2.6. Let $G$ be a torus which acts on a space $X$ such that $H_{G}^{*}(X)=P_{G} / I$ is a free $R_{G^{-}}$-module. Let $K \subset G$ be a subtorus, let $F$ and $F^{\prime}$ be connected components of the fixed space $X^{G}$ with the respective prime ideals $Q$ and $Q^{\prime}$ in $P_{G}$. Then $F$ and $F^{\prime}$ are contained in the same fixed component of $K$ iff $\left.Q\right|_{K}=\left.Q^{\prime}\right|_{K}$.

This is an immediate consequence of Lemmas 1.1 and Lemma 2.5.

We now give a more geometric interpretation of the "roots" $\left(\omega_{1 j}, \ldots, \omega_{n j}\right) \in$ $\left(H^{2}\left(B_{T}\right)\right)^{n} \cong H^{2}\left(B_{T}, \mathbf{Q}^{n}\right)$ of the associated prime ideals $P_{j} \in$ Ass $I$, if $H^{*}(X)$ is generated by elements of degree 2 . Here we assume that the coefficient field $k$ of char $k=0$ is always given by $\mathbf{Q}$. Let $n=\operatorname{edim} H^{*}(X)$. Then $H^{2}(X) \cong \mathbf{Q}^{n}$. If a compact connected Lie group $G$ acts on $X$, the map $i^{*}: H_{G}^{2}(X) \rightarrow H^{2}(X)$ is an epimorphism; in particular, $H_{G}^{2}(X)=H^{2}\left(B_{G}\right) \oplus H^{2}(X)$. (If $G$ is semisimple, $H^{2}\left(B_{G}\right)=0$.) Consider the commutative diagram

$$
\begin{array}{ccc}
H^{2}\left(X_{G} ; \mathbf{Q}\right) & \stackrel{i *}{\rightarrow} & H^{2}(X ; \mathbf{Q}) \\
j_{G} \uparrow & & \uparrow j \\
H^{2}\left(X_{G} ; \mathbf{Z}\right) & \stackrel{i_{\mathbf{Z}}^{*}}{\rightarrow} & H^{2}(X ; \mathbf{Z})
\end{array}
$$

where the vertical homomorphisms are induced by the inclusion $\mathbf{Z} \subset \mathbf{Q}$ and the horizontal maps are induced by the inclusion of the fiber. The elements in $\operatorname{Im} j_{G}$, $\operatorname{Im} j$, respectively, will be called integrally defined. Let $H^{2}(X ; \mathbf{Q})=\mathbf{Q} x_{1}$ $\oplus \cdots \oplus \mathbf{Q} x_{n}$. Then for every $x_{i}$ there exists an integral multiple $n_{i} x_{i} \in H^{2}(X ; \mathbf{Q})$ which is integrally defined. Because $i^{*}$ is surjective, there exist elements $X_{1}, \ldots, X_{n}$ $\in H^{2}\left(X_{G} ; \mathbf{Q}\right)$ with $i^{*}\left(X_{i}\right)=x_{i}$. Moreover, if we replace the $x_{i}$ by $n_{i} x_{i}$, we can choose the $X_{i}$ integrally defined too. In this way we can assume $x_{i} \in H^{2}(X ; Z)$ and $X_{i} \in H^{2}\left(X_{G} ; \mathbf{Z}\right)$. Now, assume $X$ to be a locally finite $C W$-complex and that $G$ acts on $X$ in such a way that $X_{G}$ has the homotopy type of a $C W$-complex. Note that the above assumption on $X_{G}$ is achieved, for example, if $X$ is a smooth manifold and $G$ acts smoothly on $X$. We then have the isomorphism $H^{2}(X ; \mathbf{Z})=\left[X, B_{T^{1}}\right]$, where $\left[X, B_{T^{1}}\right]$ is the set of homotopy classes of continuous mappings $f: X \rightarrow B_{T^{1}}$. Let $f_{i}$ : $X \rightarrow B_{T}, i=1, \ldots, n$, be maps in the homotopy class of $x_{i}$ respectively. Take $f$ : $X \rightarrow B_{T^{1}} \times \cdots \times B_{T^{1}}=B_{T^{n}} n$ as the map $f=f_{1} \times \cdots \times f_{n}$ and consider the bundle $\xi=f^{*} \xi_{u}$, where $\xi_{u}=\left\{T^{n} \rightarrow E_{T^{n}} \rightarrow B_{T^{n}}\right\}$ is the universal principal $T^{n}$-bundle. 
Then the first Chern class of $\xi$ is given by the $n$-tuple $c_{1}(\xi)=\left(x_{1}, \ldots, x_{n}\right) \in$ $H^{2}(X ; \mathbf{Z})^{n}$. So, we see that $c_{1}(\xi)$ is in the image of $\left(i_{\mathbf{Z}}^{*}\right)^{n}: H^{2}\left(X_{G} ; \mathbf{Z}\right)^{n} \rightarrow H^{2}(X ; \mathbf{Z})^{n}$. By Corollary 1.2 in [7] the $G$-action on $X$ can be lifted to a left bundle action on $\xi$. Let $\xi=\left\{T^{n} \rightarrow Y \rightarrow X\right\}$ and consider the bundle $\xi_{G}=\left\{T^{n} \rightarrow E_{G} \times{ }_{G} Y \rightarrow E_{G} \times_{G}\right.$ $X\}$. Then it follows from [7] ( $§ 4$, "enumeration of liftings") that the lifting can be chosen in such a way that $c_{1}\left(\xi_{G}\right)=\left(X_{1}, \ldots, X_{n}\right) \in H^{2}\left(X_{G} ; \mathbf{Z}\right)^{n}$. (In the case that $G$ is semisimple it is shown that a lifting of the $G$-action is essentially unique.) If $x \in X^{G}$ is a fixed point, $G$ acts on the fiber $T_{x}^{n}=T^{n}$ by a homomorphism $\rho_{x}$ : $G \rightarrow T^{n}$, which follows from the properties of a lifting. Moreover, $\rho_{x}$ depends only from the connected component $F$ of $X^{G}$ in which $x$ lies. If $G$ is semisimple, this homomorphism is trivial; so, if $G=S \times Z, S$ semisimple, $Z$ a central torus, we have $S \subset \operatorname{ker} \rho_{x}$; i.e., $\rho_{x}$ is defined by its restriction to $Z$. Then it is clear that $\rho_{x}$ is given by a diagonal matrix $(g \in G)$

$$
\rho_{x}(g)=\left(e^{2 \pi \sqrt{-1} \omega_{1}}, \ldots, e^{2 \pi \sqrt{-1} \omega_{n}}\right)(g)
$$

where $\omega_{i} \in H^{2}\left(B_{G} ; \mathbf{Z}\right) \cong \operatorname{Hom}\left(G, T^{1}\right) \cong \operatorname{Hom}\left(Z, T^{1}\right)$. Let $i_{x}: E_{G} \times_{G}\{x\} \subset X_{G}$ be the inclusion, take the restriction $\eta_{x}=i_{x}^{*} \xi_{G}$. Then $\eta_{x}$ is a bundle of the form

$$
\eta_{x}=\left\{T^{n} \rightarrow E_{G} \times{ }_{G} T^{n} \rightarrow B_{G}\right\},
$$

where $G$ acts on $T^{n}$ by the above homomorphism. From the Borel-Hirzebruch description of characteristic classes it follows that the first Chern class $c_{1}\left(\eta_{x}\right)$ is given by the $n$-tuple $\left(\omega_{1}, \ldots, \omega_{n}\right) \in H^{2}\left(B_{T} ; \mathbf{Z}^{n}\right)$. So, by naturality, we obtain $i_{x}^{*}\left(X_{1}, \ldots, X_{n}\right)=\left(\omega_{1}, \ldots, \omega_{n}\right)$. Now, assume $G$ a torus, write $H_{G}^{*}(X)=$ $R_{G}\left[X_{1}, \ldots, X_{n}\right] / I$. Then it follows from Theorem 2.1(i) that the associated prime ideal $P$ of the fixed space component $F$ is given by $P=\left(X_{1}-\omega_{1} \otimes \mathrm{z} 1, \ldots, X_{n}-\right.$ $\left.\omega_{n} \otimes \mathbf{z} 1\right)$.

We collect our results in the following theorem.

THEOREM 2.2. Let $X$ be a locally finite $C W$-complex such that $H^{*}(X)$ is generated as a graded Q-algebra by finitely many elements of degree two. Let $G$ be a torus which acts in such a way that $X_{G}$ has the homotopy type of a $C W$-complex. Take a generator system $y_{1}, \ldots, y_{n}$ of $H^{*}(X)$ and write $H_{G}^{*}(X)=R_{G}\left[Y_{1}, \ldots, Y_{n}\right] / I$ such that the "edge"-homomorphism is given by $i^{*}\left(Y_{i}\right)=y_{i}$. Then there exist integral multiples $X_{i}=m_{i} Y_{i}, x_{i}=m_{i} y_{i}$ which are integrally defined. Moreover there exists a $T^{n}$-principal bundle $\xi$ on $X$ with $c_{1}(\xi)=\left(x_{1}, \ldots, x_{n}\right)$. The $G$ action can be lifted in $\xi$ such that $c_{1}\left(\xi_{G}\right)=\left(X_{1}, \ldots, X_{n}\right)$. Let $F \subset X^{G}$ be a connected component of the fixed space, take $x \in F$, let $\left(\omega_{1}, \ldots, \omega_{n}\right)$ be the weights of the homomorphism $\rho_{x}: G \rightarrow T^{n}$ in the fiber $T_{x}^{n}$ over $x$ in $\xi$. Then the prime ideal $P \subset R_{G}\left[Y_{1}, \ldots, Y_{n}\right]$ of the component $F$ is given by $P=\left(Y_{1}-\omega_{1} / m_{1} \otimes 1, \ldots, Y_{n}-\omega_{n} / m_{n} \otimes 1\right)$.

Thus we see that up to an integral factor the "roots" $\omega_{i j}$ of a prime ideal $P_{j} \in$ Ass $I$ can be interpreted as weights of a local representation in a $T^{n}$-principal bundle $\xi$ which is intimately connected with the cohomology ring structure of $X$. 
3. The rank of a prime ideal and the torus symmetry degree. An ( $n$-ary) quadratic form over a field $k$ is a polynomial $Q$ in $n$ variables over $k$, which is homogeneous of degree two. It has the form

$$
Q\left(x_{1}, \ldots, x_{n}\right)=\sum_{i, j=1}^{n} a_{i j} x_{i} x_{j} \in k\left[x_{1}, \ldots, x_{n}\right]
$$

where it is no loss of generality to assume that the matrix $M_{Q}=\left(a_{i j}\right)$ is symmetric. In matrix notation we have

$$
Q\left(x_{1}, \ldots, x_{n}\right)=\left(x_{1}, \ldots, x_{n}\right) \cdot M_{Q} \cdot\left(\begin{array}{l}
x_{1} \\
\vdots \\
x_{n}
\end{array}\right)=X^{t} \cdot M_{Q} \cdot X,
$$

Let $Q$ and $Q^{\prime}$ be $n$-ary quadratic forms. We say that $Q$ is equivalent to $Q^{\prime}$ if there exists an invertible matrix $C \in \mathrm{GL}_{n}(k)$ such that $Q(x)=Q^{\prime}(C x)$. This means that there exists a nonsingular homogeneous linear substitution of the variables $x_{1}, \ldots, x_{n}$, which takes the form $Q^{\prime}$ of the form $Q$. Since

$$
Q^{\prime}(C x)=(C x)^{t} \cdot M_{Q^{\prime}} \cdot(C x)=X^{t}\left(C^{t} M_{Q}, C\right) x
$$

the equivalence condition $Q(x)=Q^{\prime}(C x)$ stipulated above amounts to a matrix equation

$$
M_{Q}=C^{t} \cdot M_{Q^{\prime}} \cdot C
$$

The following is fundamental in the theory of quadratic forms.

THEOREM (DIAgONALIZABILITY OF QUADRATIC FORMS). Let $k$ be a field, char $k \neq 2$, let $Q$ an $n$-ary quadratic form over $k$. Then $Q$ is equivalent (over $k$ ) to a diagonal form $d_{1} X_{1}^{2}+\cdots+d_{n} X_{n}^{2}, d_{i} \in k$.

For a proof see e.g. the book of T. Y. Lam [13, p. 10]. If $X$ is a space of $q$-type, $H^{*}(X)=\mathbf{Q}\left[x_{1}, \ldots, x_{n}\right] / I_{0}, Q \in I_{0}$ a definite quadratic form, then it follows from the above theorem that we can choose new homogeneous generators $x_{1}^{\prime}, \ldots, x_{n}^{\prime}$ of $H^{*}(X)$ such that $Q$ takes a diagonal form. Moreover, the entries of the corresponding matrix $M_{Q}$ are then all either positive or negative. So, we can assume that $Q$ has the form $Q\left(x_{1}, \ldots, x_{n}\right)=d_{1} x_{1}^{2}+\cdots+d_{n} x_{n}^{2}, d_{i}<0$. The following lemma is fundamental for our considerations.

LEMMA 3.1. Let $k$ be a field, char $k \neq 2$, let $A_{0}=k\left[y_{1}, \ldots, y_{n}\right] / I_{0}$ be a graded $k$-algebra, $\operatorname{deg} y_{i}=2, i=1, \ldots, n$. Let $R=k\left[t_{1}, \ldots, t_{r}\right]$ be a positively graded polynomial ring, $\operatorname{deg} t_{j} \equiv O(2)$ and let $A$ be a graded flat $R$-algebra such that $A \otimes_{R} k=A_{0}$. If there exists a definite quadratic form $Q$ in $I_{0}$, then homogeneous generators $x_{1}, \ldots, x_{n}$ of $A_{0}$ and $X_{1}, \ldots, X_{n}$ of $A$ can be found such that

(i) $X_{i} \otimes_{R} 1=x_{i}$.

(ii) $Q$ is diagonal in the variables $x_{i}$.

(iii) If we write $A=R\left[X_{1}, \ldots, X_{n}\right] / I$, then there exists an element $\tilde{Q}-\beta$ in I with $\tilde{Q}=Q\left(X_{1}, \ldots, X_{n}\right)$ and $\beta \in R_{+}, \operatorname{deg} Q=\operatorname{deg} \beta$.

Proof. Without loss of generality we assume $Q$ to be diagonal to the variables $x_{i}=y_{i}$; i.e., $Q\left(y_{1}, \ldots, y_{n}\right)=d_{1} y_{1}^{2}+\cdots+d_{n} y_{n}^{2}$. After Theorem 1.1 we can write $A=R\left[Y_{1}, \ldots, Y_{n}\right] / J$, such that there exists a homogeneous element of the form 
$Q\left(Y_{1}, \ldots, Y_{n}\right)-r, r \in R_{+} R[Y]$, in $J$. By graduation reasons, $r$ must take the form $r=\sum_{i=1}^{n} \lambda_{i} Y_{i}+\gamma$, where the $\lambda_{i}, \gamma$ are homogeneous elements of $R$. After changing the generators $Y_{i}$ in $X_{i}=Y_{i}-\lambda_{i} / 2 d_{i}$ the linear terms in the $Y_{i}$ disappear and the above expression takes the form $Q\left(X_{1}, \ldots, X_{n}\right)-\beta$, where $\beta$ is given by $\beta=$ $\sum_{i=1}^{n}\left(\lambda_{i} / 4 d_{i}^{2}\right)+\gamma$. Because we have $Y_{i} \otimes_{R} 1=y_{i}=x_{i}$ it follows that $X_{i} \otimes_{R} 1=x_{i}$.

If $X$ is a space of $q$-type and a compact connected Lie group $G$ acts on $X$, then $A_{0}=H^{*}(X)$ and $A=H_{G}^{*}(X)$ satisfy the hypothesis of Lemma 3.1. (Recall that we assumed char $k=0$.) We call a presentation $H_{G}^{*}(X)=R_{G}\left[X_{1}, \ldots, X_{n}\right] / I$ normalized, if the generators $X_{i}$ and $x_{i}=X_{i} \otimes 1$ have the property of Lemma 3.1. It is clear that the generators can be chosen as integrally defined.

Henceforth we shall always assume that the ground field is given by $\mathbf{Q}$, the field of rational numbers. Let $X$ be a space and $G$ a compact connected Lie group which acts on $X$. Then the action is called cohomologically effective (c-effective) if $H^{*}\left(X, X^{S}\right) \neq 0$ for every subtorus $S \subset G$ with $\operatorname{dim} S>0$.

Lemma 3.2. Let $X$ be a space of q-type, let $G$ be a connected compact Lie group which acts c-effectively on $X$. Let $H_{G}^{*}(X)=P_{G} / I$ be a normalized presentation and let $\tilde{Q}-\beta \in I$ be the element corresponding to the definite quadratic form $Q \in I_{0}$. Then $\beta$ does not vanish. The same is true if $G$ is a torus which acts on $X$ such that $H^{*}\left(X, X^{G}\right) \neq 0$.

Proof. Let $T \subset G$ be the maximal torus. Then by Theorem 2.2 we have $H_{T}^{*}(X) \cong H_{G}^{*}(X) \otimes_{R_{G}} R_{T}$. So, if $J \subset P_{T}$ is the ideal generated by the elements of $I$, we have $H_{T}^{*}(X) \cong P_{T} / J$. Therefore we can consider $\tilde{Q}-\beta$ as an element of $J$. Now by the hypothesis $T$ acts such that $H^{*}\left(X, X^{T}\right) \neq 0$. Suppose $\beta=0$. Then $V(I) \subset$ $V(\tilde{Q})$ of varieties in $\operatorname{Spec} P_{T}$. Because $Q$ is definite, $V(\tilde{Q})$ is the variety given by $X_{1}=X_{2}=\cdots=X_{n}=0$; i.e., $V(\tilde{Q})=V(P), P=\left(X_{1}, \ldots, X_{n}\right)$. So it follows that $V(I)=V(P)$; i.e., $I$ is $p$-primary. Then the fixed space $X^{T}$ must be connected. By Lemma 2.1 it follows that $H^{*}\left(X, X^{T}\right)=0$, a contradiction.

Definition 3.1. Let $G$ be a torus, let $P \subset P_{G}=R_{G}\left[X_{1}, \ldots, X_{n}\right]$ a prime ideal, $P=\left(X_{1}-\omega_{1}, \ldots, X_{n}-\omega_{n}\right), \omega_{i} \in H^{2}\left(B_{G}\right)$, then rk $P$ is the rank of the matrix $\omega=\left(\omega_{1}, \ldots, \omega_{n}\right)$.

Lemma 3.3. Let $X$ be a space of q-type, let $G$ be a torus of rank $G=m$ which acts on $X$, let $H_{G}^{*}(X)=P_{G} / I$ be a normalized presentation. Let $\tilde{Q}-\beta \in I$ be the elements considered above. If $P \in$ Ass $I$ is a prime ideal with $P=\left(X_{1}-\omega_{1}, \ldots, X_{n}\right.$ - $\left.\omega_{n}\right)$, then $\beta=Q\left(\omega_{1}, \ldots, \omega_{n}\right)$. If $G$ acts c-effectively, then $\operatorname{rk} P=\operatorname{rk} G=m$.

Proof. Because $P$ is an associated prime ideal of $I$, we have $\tilde{Q}-\beta \in P$; i.e., $\beta=Q\left(\omega_{1}, \ldots, \omega_{n}\right)$. Let $V \subset L T$ be the $Q$-vector space of the rational points of the real Lie algebra of $T$. Then $Q \circ \omega=Q\left(\omega_{1}, \ldots, \omega_{n}\right)$ is a quadratic form on $V$. Let $W \subset V$ be a subvector-space. Then there exists a subtorus $S \subset G$ whose Lie algebra $L S$ is spanned by $W$. If $G$ acts c-effectively it follows from Lemmas 2.3 and 3.2 that the restriction $\left.Q \circ \omega\right|_{W}$ does not vanish. This shows $Q \circ \omega$ to be a definite quadratic form on $V$. But $Q$ is definite, so the linear map $\omega=\left(\omega_{1}, \ldots, \omega_{n}\right): V \rightarrow \mathbf{Q}^{n}$ must be injective; i.e., $\mathrm{rk} \omega=\mathrm{rk} P=\mathrm{rk} G$. 
As a corollary of the lemma we obtain

THEOREM 3.1. Let $X$ be a space of q-type. If a torus $G$ acts c-effectively on $X$, then rk $G \leqslant \operatorname{edim} H^{*}(X)$.

ProOF. The number of generators has not been affected by the choices we have made. Thus we can assume $n=\operatorname{edim} H^{*}(X)$. But rk $\omega$ cannot be higher than $n$. So, it follows that $\mathrm{rk} G=\operatorname{rk} \omega \leqslant \operatorname{edim} H^{*}(X)$.

Denote by $\operatorname{cd}_{\mathbf{Q}} Y$ the $\mathbf{Q}$-cohomology dimension of a connected space $Y$. If $Y$ is disconnected, let $\operatorname{cd}_{\mathrm{Q}} Y$ be the maximum of $\operatorname{cd}_{\mathrm{Q}} Y_{i}$ over all its connected components, if it exists. If it does not exist, put $\operatorname{cd}_{\mathrm{Q}} Y=\infty$. If $Y$ is a manifold, then $\operatorname{cd}_{\mathbf{Q}} Y \leqslant \operatorname{dim} Y$. If $Y$ is a closed connected compact oriented manifold, then $\operatorname{cd}_{\mathbf{Q}} Y=$ $\operatorname{dim} Y$.

THEOREM 3.2 (THEOREM 1). Let $X$ be a closed connected compact oriented manifold of q-type. Then we have the inequality $T_{t}(X) \leqslant \operatorname{edim} H^{*}(X)$.

Proof. If a torus $G$ acts effectively on $X$, then let $S \subset G$ be a subtorus s.t. $H^{*}\left(X, X^{S}\right)=0$. It follows that $\operatorname{cd}_{\mathbf{Q}} X=\operatorname{cd}_{\mathbf{Q}} X^{S}$. By the above remark this implies $\operatorname{dim} X=\operatorname{dim} X^{S}$; i.e., $S$ acts trivially and the action cannot be effective. This shows that an effective action of $G$ on $X$ is also c-effective. By Theorem 3.1 it follows that rk $G \leqslant \operatorname{edim} H^{*}(X)$.

As an application to homogeneous spaces we obtain the following result.

THEOREM 3.3. Let $G$ be a compact connected semisimple Lie group and let $T \subset G$ be a maximal torus. Then $T_{t}(G / T)=\mathrm{rk} G$.

Proof. By the remarks made in the introduction $X=G / T$ is a space of $q$-type. Then it satisfies the hypotheses of Theorem 3.2. So, we have $T_{t}(X) \leqslant \operatorname{edim} H^{*}(X)$ $=$ rk $G$. Since $T$ acts effectively on $X$ by left translations, it follows that $T_{t}(X)=$ rk $G$.

4. Smoothness, embedding dimension, and the cohomology of fixed spaces. The existence of a definite quadratic form in the defining ideal of $H^{*}(X)$ can also be used to get more information on the cohomology of the fixed space $X^{G}$ of a torus action. We begin with the following lemma.

LEMMA 4.1. Let $k$ be a field, char $k \neq 2$. Let $V$ be a vector space over $k, \operatorname{dim} V=n$. Denote by $\mathcal{O}_{V}=k\left[x_{1}, \ldots, x_{n}\right]$ the ring of regular functions on $V$ and assume $Q \in \mathcal{O}_{V}$ to be a definite quadratic form. Let $\beta \in k-\{0\}$, and consider the variety $Y=$ $V(Q-\beta)=\operatorname{Spec}_{V} /(Q-\beta)$. Then $Y$ is smooth in every $k$-valued point $y \in Y$ (if it has one ); i.e., $\operatorname{edim} \mathcal{O}_{Y, y}=\operatorname{dim} \mathcal{O}_{Y, y}=n-1$. Here $\mathcal{O}_{Y, y}$ is the local ring of $Y$ in $y$.

Proof. Let $\mathcal{O}_{Y}=\mathcal{O}_{Y} /(Q-\beta)$. Then the exact sequence in Matsumura (p. 187, Theorem 58) gives us for the Kählerian module $\Omega_{Y \mid k}$

$$
\Omega_{Y \mid k}=\frac{\sum_{i=1}^{n} \mathcal{O}_{Y} d x_{i}}{\left(\omega_{1} d x_{1}+\cdots+\omega_{n} d x_{n}\right)},
$$


where $\omega_{i}=\partial Q / \partial x_{i}$. By the Euler identity we have $Q=\frac{1}{2} \sum_{i=1}^{n} x_{i} \omega_{i}$. So it follows from the definiteness of $Q$ that the matrix $\omega=\left(\omega_{1}, \ldots, \omega_{n}\right)$ has maximal rank; in particular, the ideal $\left(\omega_{1}, \ldots, \omega_{n}\right)$ has the zero set $V\left(\omega_{1}, \ldots, \omega_{n}\right)=\{0\}$. Because of $\beta \neq 0$, it follows that $0 \notin Y$. Let $y \in Y$ be a $k$-valued point with maximal ideal $\mathfrak{m}$. Then $\left(\omega_{1}, \ldots, \omega_{n}\right) \not \subset \mathrm{m}$. Consequently, there must exist an $\omega_{j}$, say $\omega_{1}$, with $\omega_{1} \in \mathcal{O}_{Y}$ - $m$. Therefore we obtain for the Kählerian module localized at $m$ :

$$
\Omega_{Y \mid k} \otimes_{\mathcal{O}_{Y}} \mathcal{O}_{Y, \mathrm{~m}}=\frac{\sum_{i=1}^{n} \mathcal{O}_{Y, \mathrm{~m}} d x_{i}}{d x_{1}+\sum_{i=2}^{n} \omega_{i} / \omega_{1} d x_{i}}=\sum_{i=2}^{n} \mathcal{O}_{Y, \mathrm{~m}} d x_{i},
$$

i.e., $\Omega_{Y \mid k}$ is locally free around $y$ of rank $n-1$. So, the cotangential space $\mathrm{m} / \mathrm{m}^{2}$ has dimension $n-1$, which is equal to the local dimension of $Y$ in $y$; i.e., $Y$ is smooth in $y$.

Let $X$ be a space of type $q$, and let $G$ be a circle which acts on $X$. Then after Lemma 1.2 we have for the fixed set $X^{G}$ :

$$
H^{*}\left(X^{G} ; K_{\eta}\right) \cong H_{G}^{*}(X) \otimes_{R_{G}} K_{\eta},
$$

where $K_{\eta}$ is the residue field of $R_{G}=\mathbf{Q}[t]$ in the point $t=1$. If we write $H^{*}(X)=P_{G} / I, P_{G}=R_{G}\left[X_{1}, \ldots, X_{n}\right]$, then we have

$$
H^{*}\left(X^{G} ; K_{\eta}\right) \cong K_{\eta}\left[X_{1}, \ldots, X_{n}\right] / I_{\eta},
$$

where the ideal $I_{\eta}$ is generated by the evaluations of the elements $F \in I$ at $t=1$. The $K_{\eta}$-algebra $H_{G}^{*}(X) \otimes_{R_{G}} K_{\eta}$ is a semilocal algebra, all of whose residue fields are isomorphic to $K_{\eta} \cong \mathbf{Q}$. Obviously there is a natural bijection between the local components of $H_{G}^{*}(X) \otimes_{R_{G}} K_{\eta}$ and the set of connected components $\pi_{0} X^{G}$ of the fixed space. If the equivariant cohomology has been written in a normalized form, then there exists in $I$ an element $Q-\beta t^{2}$, where $Q$ is the definite quadratic form. Its evaluation in $t=1$ is given by $Q-\beta \in I_{\eta}$. So we see that the zero-dimensional variety $V\left(I_{\eta}\right)$ is a closed subvariety of the ellipsoid $Y=V(Q-\beta)$ in $\operatorname{Spec} K_{\eta}\left[X_{1}, \ldots, X_{n}\right]$.

THEOREM 4.1 (THEOREM 2). Let $X$ be a space of q-type, let $G$ be a torus which acts on $X$ such that $H^{*}\left(X, X^{G}\right) \neq 0$. Then every connected component $F$ of $X^{G}$ is a space of q-type with $\operatorname{edim} H^{*}(F)<\operatorname{edim} H^{*}(X)$.

Proof. As we have seen in Theorem 2.1, $H_{G}^{*}(F)$ is a quotient of $H_{G}^{*}(X)$. Then it follows that $H^{*}(F)$ is a quotient of $H^{*}(X)$. Denote by $\varphi: H^{*}(X) \rightarrow H^{*}(F)$ the surjective homomorphism induced by the inclusion $F \subset X$. Let $H^{*}(X)=$ $\mathbf{Q}\left[x_{1}, \ldots, x_{n}\right] / I_{0}$ and $H^{*}(F)=\mathbf{Q}\left[y_{1}, \ldots, y_{m}\right] / I_{F}$. Then there exists a graded epimorphism $\Phi$ of the polynomial rings such that the following diagram commutes:

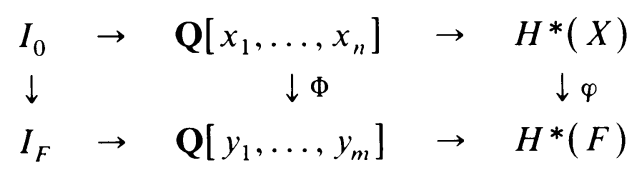

By graduation reasons $\Phi$ is given by linear polynomials; i.e., $\Phi$ is induced by a linear inclusion $V^{m} \subset W^{n}$ of $\mathbf{Q}$-vector spaces. Let $Q_{F}=\Phi(Q) \in I_{F}$. Then $Q_{F}$ is simply the restriction $Q_{F}=\left.Q\right|_{V} m$. It follows that $Q_{F}$ is also definite. 
It remains to prove the statement about the embedding dimension. Because $G$ acts on $X$ with finitely many orbit types, there exists a circle $G^{\prime} \subset G$ with $X^{G}=X^{G^{\prime}}$. Therefore we can assume that $G$ is a circle. We write $H_{G}^{*}(X)=P_{G} / I$ in a normalized form. The ideal $I_{\eta} \subset K_{\eta}\left[X_{1}, \ldots, X_{n}\right]$ contains the element $Q-\beta, \beta \in$ $K_{\eta}-\{0\}$. Let $Z=\operatorname{Spec} K_{\eta}\left[X_{1}, \ldots, X_{n}\right] / I_{\eta}$, and let $z \in Z$ be the $K_{\eta}$-valued point corresponding to the fixed space component $F$. Then $H^{*}\left(F ; K_{\eta}\right)=\mathcal{O}_{Z, z}$. But $z \in Y=\operatorname{Spec} K_{\eta}\left[X_{1}, \ldots, X_{n}\right] /(Q-\beta)$ and $\mathcal{O}_{Z, z}$ is a quotient of $\mathcal{O}_{Y, z}$. Therefore it follows that $\operatorname{edim} \mathcal{O}_{Z, z} \leqslant \operatorname{edim} \mathcal{O}_{Y, z}$. By Lemma 4.1 it follows that edim $H^{*}\left(F ; K_{\eta}\right)$ $\leqslant n-1$. Because $n$ can be chosen as $n=\operatorname{edim} H^{*}(X)$ and $K_{\eta} \cong \mathbf{Q}$ this implies edim $H^{*}(F)<\operatorname{edim} H^{*}(X)$.

ObSeRvation. Let $X$ be a space of $q$-type with edim $H^{*}(X)=1$. Then $X \sim{ }_{\mathrm{Q}} S^{2}$. Proof. We have $H^{*}(X) \cong \mathrm{Q}[x] / I_{0}$ with $Q \in I_{0}$ a definite quadratic form. Then $Q=d \cdot x^{2}, d \neq 0$. Because $\mathbf{Q}[x]$ is a principal ideal domain this shows $H^{*}(X) \cong$ $\mathbf{Q}[x] /\left(x^{2}\right)$.

THEOREM 4.2 (THeOREM 3). Let $X$ be a space of q-type with edim $H^{*}(X)=2$. If a torus $G$ acts on $X$ with fixed space $X^{G}$ such that $H^{*}\left(X, X^{G}\right) \neq 0$, then

$$
X^{G} \sim{ }_{\mathrm{Q}} S^{2}+\cdots+S^{2}+\text { isolated points. }
$$

Proof. If $F$ is a connected component of $X^{G}$ then it follows from Theorem 4.1 that edim $H^{*}(F) \leqslant 1$. If edim $H^{*}(F)=1$, then, by the above observation it follows that $F \sim{ }_{\mathrm{Q}} S^{2}$. If edim $H^{*}(F)=0$, then $F \sim{ }_{\mathrm{Q}}$ pt.

OBSERVATION. Let $X$ be a space whose cohomology is generated as a $\mathbf{Q}$-algebra by finitely many elements of degree 2 . Assume a torus $G$ acts on $X$. Then the action is c-effective iff $\operatorname{cd}_{\mathbf{Q}} X^{S}<\operatorname{cd}_{\mathbf{Q}} X$ for all subtori $S \subset G$.

Proof. If $G$ acts c-effectively on $X$, then $H^{*}\left(X, X^{S}\right) \neq 0$ for any subtorus $S \subset G, S \neq e$. Let $Y$ be a connected component of $X^{S}$ with $\operatorname{cd}_{\mathbf{Q}} Y=\operatorname{cd}_{\mathbf{Q}} X^{S}$. Then the restriction map $H^{*}(X) \rightarrow H^{*}(Y)$ induced by the inclusion $Y \subset X$ has a nontrivial kernel $J$. Let $t \in H^{*}(X)$ be a top-dimensional element. Then $t$ is in the socle of the Q-algebra $H^{*}(X)$, so it follows by the properties of the socle that $t \in J$. Because the restriction map is surjective this shows $\operatorname{cd}_{\mathbf{Q}} Y<\operatorname{cd}_{\mathbf{Q}} X$; i.e., $\operatorname{cd}_{\mathbf{Q}} X^{S}<$ $\operatorname{cd}_{\mathbf{Q}} X$. If, conversely, $\operatorname{cd}_{\mathbf{Q}} X^{s}<\operatorname{cd}_{\mathbf{Q}} X$, then trivially, $H^{*}\left(X, X^{S}\right) \neq 0$.

THEOREM 4.3. Let $X$ be a space of q-type which is a $\mathbf{Q}$-cohomology manifold. Let $G$ be a torus of $\operatorname{rank} G=k \leqslant n=\operatorname{edim} H^{*}(X)$, which acts c-effectively on $X$. Then edim $H^{*}(F) \leqslant n-k$ for every connected component $F$ of $X^{G}$.

Proof. Let $x \in F$ and denote by $\Omega_{X}=\left\{ \pm w_{i} ; k_{i}\right\} \cup\{0 ; r\}$ the topological weight system of the action in the component $F$. (For these and similar concepts we refer to the monograph of W. Y. Hsiang [12], in particular see Theorem (V.I').) If $K \subset G$ is a subtorus, denote by $F_{x}(K)$ the fixed space component of $K$ which contains $x$. Then the $w_{i}$ are precisely those corank-1-subtori with $\operatorname{cd}_{\mathbf{Q}} F_{x}\left(w_{i}\right)>\operatorname{cd}_{\mathbf{Q}} F$. Let $N=\cap w_{i}$. Then it follows from the Borel formula (or the above-cited theorem (ii)) that $\operatorname{cd}_{\mathbf{Q}} F_{x}(N)=\mathrm{cd}_{\mathbf{Q}} X$. Because the action is assumed to be c-effective, this shows $N=e$, using the last observation. So, it follows that there exist $k$ weights $w_{1}, \ldots, w_{k}$ in the weight system with $w_{1} \cap \cdots \cap w_{k}=e$. Let $G_{j}=w_{1} \cap \cdots \cap w_{j}, 1 \leqslant j \leqslant k$. 
Then again by Theorem (V.I') we have a chain of true inclusions of subspaces

$$
F \subset F_{x}\left(G_{1}\right) \subset F_{x}\left(G_{2}\right) \subset \cdots \subset F_{x}\left(G_{k-1}\right) \subset F_{x}\left(G_{k}\right) \sim{ }_{\mathrm{Q}} X .
$$

Moreover, we have $F_{x}\left(G_{j}\right)=F_{x}\left(G_{j+1} / G_{j}, F_{x}\left(G_{j+1}\right)\right)$ and $\operatorname{cd}_{\mathbf{Q}} F_{x}\left(G_{j}\right)<\operatorname{cd}_{\mathbf{Q}} F_{x}\left(G_{j+1}\right)$. But then, by the last observation and Theorem 4.1 it follows that edim $H^{*}\left(F_{x}\left(G_{j}\right)\right)$ $<\operatorname{edim} H^{*}\left(F_{x}\left(G_{j+1}\right)\right), j=0, \ldots, k-1$, where we have put $G_{0}=G$. This implies $\operatorname{edim} H^{*}(F) \leqslant n-k$.

The following is a corollary of this fact.

THEOREM 4.4. Let $X$ be a space of q-type which is a Q-cohomology manifold, $n=\operatorname{edim} H^{*}(X)$. Let $G$ be a torus of rank $k \leqslant n$ which acts $c$-effectively on $X$. Then

(i) If $k=n-1$, the fixed space decomposes as $X^{G} \sim{ }_{\mathbf{Q}} S^{2}+\cdots+S^{2}+$ isolated points.

(ii) If $k=n, X^{G} \sim \mathbf{Q}$ isolated points.

Proof. If $k=n-1$, then every component $F$ of $X^{G}$ has edim $H^{*}(F) \leqslant 1$. By the same argument as in the proof of Theorem 4.2 we obtain $F \sim{ }_{\mathrm{Q}} S^{2}$ or $F \sim{ }_{\mathrm{Q}} \mathrm{pt}$.

If $k=n$, then $\operatorname{edim} H^{*}(F)=0$; i.e., $H^{*}(F) \cong \mathbf{Q}$ or $F \sim_{\mathrm{Q}}$ pt.

A little remark can also be made on the topological weight systems in the different fixed space components if the acting torus $G$ has the maximal possible rank; i.e. rk $G=\operatorname{edim} H^{*}(X)$.

LEMMA 4.2. Let $X$ be a space of q-type which is a Q-cohomology manifold, $n=\operatorname{edim} H^{*}(X)$. Let $G$ be a torus of $\mathrm{rk} G=n$ which acts c-effectively on $X$. Let $x \in F, F$ a connected component of $X^{G}$, then the topological weight system $\Omega_{x}$ is given by $\Omega_{x}=\left\{ \pm w_{i} ; 1\right\}$ where the number of weights is equal to $\frac{1}{2} \mathrm{~cd}_{\mathbf{Q}} X$.

Proof. Let $w$ be a weight in $\Omega_{x}$. By an analogous argument as in the proof of Theorem 4.3 it follows that edim $H^{*}\left(F_{x}(w)\right)=1$. But then $F_{x}(w) \sim{ }_{\mathbf{Q}} S^{2}$ and the multiplicity of the weight is equal to 2. By the Borel formula it follows that the number of weights is equal to $\frac{1}{2} \mathrm{~cd}_{\mathbf{Q}} X$.

5. Applications to the actions of semisimple and arbitrary compact Lie groups. If a compact semisimple Lie group $G$ acts on a space $X$, then the Weyl group $W G=$ $N T / T$ acts in a natural way on the fixed space $X^{T}$ of the induced action of the maximal torus $T$ of $G$. This induces an action on the set $\pi_{0} X^{T}$ of connected components of $X^{T}$. If $X$ is a space whose $\mathbf{Q}$-cohomolgy is generated as a $\mathbf{Q}$-algebra by even-dimensional elements, this action is reflected in an action on $H_{T}^{*}(X) \cong$ $H_{G}^{*}(X) \otimes_{R_{G}} R_{T}$. In particular, $W G$ acts on Ass $I_{T}$, the set of associated prime ideals of the $P_{T}$-module $H_{T}^{*}(X)=P_{T} / I_{T}$. If $P=\left(X_{1}-\omega_{1}, \ldots, X_{n}-\omega_{n}\right) \subset P_{T}$ is such a prime ideal, $\omega_{i} \in R_{T}$, then the action is given by $P^{\sigma}=\left(X_{1}-\omega_{1}^{\sigma}, \ldots, X_{n}-\omega_{n}^{\sigma}\right)$, $\sigma \in W G$. If $X$ is a space of $q$-type, then it is easy to see that a normalized form of $H_{G}^{*}(X)$ gives rise to a normalized form of $H_{T}^{*}(X)$ which is $W G$-invariant.

THEOREM 5.1. Let $X$ be a space of $q$-type, let $G$ be a compact semisimple Lie group which acts c-effectively on $X$, and let $T \subset G$ be a maximal torus of $G$. Then $W G=N T / T$ acts freely on $\pi_{0} X^{T}$. 
Proof. Assume $H_{G}^{*}(X)=P_{G} / I_{G}$ has been written in a normalized form. By tensoring the presentation with $R_{T}$ over $R_{G}$ we obtain a normalized form of $H_{T}^{*}(X)=P_{T} / I_{T}$, where $I_{T}$ is the ideal generated by the elements of $I_{G}$ using the canonical embedding $P_{G} \subset P_{T}$. By the above remark it suffices to consider the action of $W G$ on Ass $I_{T}$. Let $P \in$ Ass $I_{T}, P=\left(X_{1}-\omega_{1}, \ldots, X_{n}-\omega_{n}\right)$. Then the isotropy subgroup $W G_{P}$ of the prime ideal $P$ is given by $W G_{P}=\left\{\sigma \in W G ; \omega_{i}=\omega_{i}^{\sigma}\right.$, $i=1, \ldots, n\}$ as one sees as follows: Let $\sigma \in W G$ with $P=P^{\sigma}$. Then, by graduation reasons, we have

$$
X_{i}-\omega_{i}=\sum_{j=1}^{n} \lambda_{i j}\left(X_{j}-\omega_{j}^{\sigma}\right),
$$

with $\left(\lambda_{i j}\right) \in M(n, n ; \mathbf{Q})$. Because there are no relations between the $X_{i}$ and the generators of $R_{G}$, it follows that

$$
X_{i}=\sum_{j=1}^{n} \lambda_{i j} X_{j}
$$

and by the linear independence of the $X_{i}$ this implies $\lambda_{i j}=0, i \neq j, \lambda_{i i}=1$ and therefore $\omega_{i}=\omega_{i}^{\sigma}, i=1, \ldots, n$. Thus we have

$$
W G_{P}=\bigcap_{i=1}^{n} W G_{\omega_{i}},
$$

where $W G_{\omega_{1}}$ is the isotropy group of $\omega_{i} \in H^{2}\left(B_{T} ; \mathbf{Q}\right) \cong \operatorname{Hom}_{\mathbf{Q}}(L T, \mathbf{Q})$ with respect to the natural action of $W G$ on the dual of the Lie algebra $L T$ of $T$. Denote by $\Delta G$ the root system of $G$, let $\alpha \in \Delta G$ be a (not necessarily positive) root and let $S_{\alpha}$ be the reflection in the hyperplane $H_{\alpha}$ perpendicular to $\alpha$. Then $W G_{\omega_{i}}$ is generated by exactly those $S_{\alpha}$ with $\left(\alpha, \omega_{i}\right)=0$, where $($,$) is the bilinear form induced by the$ Killing form on the dual of $L T$. Now, the intersection of reflection groups is again a reflection group. So $W G_{P}$ is generated as a reflection group by the $S_{\alpha}$ with $\left(\alpha, \omega_{i}\right)=0$ for all $i, i=1, \ldots, n$. By Lemma 3.3 we have $\operatorname{rk}\left(\omega_{1}, \ldots, \omega_{n}\right)=\mathrm{rk} T$. But the inner product $($,$) is nondegenerate. It follows that a root which is perpendicular$ to all $\omega_{i}$ must vanish; i.e., $W G_{P}=1$. Therefore the group $W G$ acts freely on Ass $I_{T}$ and so it acts freely on $\pi_{0} X^{T}$.

As a corollary we obtain the following divisibility condition.

THEOREM 5.2 (THEOREM 4). Let $X$ be a space of q-type, and let $G$ be a semisimple compact connected Lie group which acts c-effectively on $X$. Then the Euler number $\chi(X)$ of $X$ is divided by the order $|W G|$ of the Weyl group $W G$ of $G$.

Proof. Denote by $\pi=W G \backslash \pi_{0} X^{T}$ the discrete quotient of the $W G$-action on $\pi_{0} X^{T}$. We then have

$$
X^{T}=\sum_{j \in \pi} W G \cdot F_{j},
$$

where $F_{j}$ is a connected component of $X^{T}$ in the equivalence class of $j$. Because $W G$ acts freely on $\pi_{0} X^{T}$ it follows that $W G \cdot F_{j}=W G \times F_{j}$. Thus we have

$$
\chi\left(X^{T}\right)=|W G| \cdot\left(\sum_{j \in \pi} \chi\left(F_{j}\right)\right) .
$$


The classical result $\chi(X)=\chi\left(X^{T}\right)$ then gives

$$
\chi(X)=|W G| \cdot\left(\sum_{j \in \pi} \chi\left(F_{j}\right)\right) \text {. Q.E.D. }
$$

An immediate corollary of Theorem 5.4 is the following nice observation which is difficult to find in the literature although it is surely known. (Probably it is involved in the work of Dynkin $[3,4]$.)

ObSERVATION. If $G$ is a compact semisimple Lie group, $H \subset G$ a closed connected semisimple subgroup, then $|W G|=|W H| \cdot s, s$ a natural number.

Proof. Let $X=G / T, T \subset G$ a maximal torus. If $Z(H)$ is the center of $H$, then $H^{\prime}=H / Z(H)$ acts effectively on $X$ by left translations. As in the proof of Theorem 3.2 it follows that $H^{\prime}$ acts c-effectivley on $X$. But $X$ is a space of $q$-type with $\chi(X)=|W G|$. Then the result follows from the above theorem together with the fact that $W H=W H^{\prime}$.

Let $X$ be a space of $q$-type which is a locally finite $C W$-complex. Assume that a compact connected Lie group $G$ acts on $X$ in such a way that $X_{G}$ has the homotopy type of a $C W$-complex. Assume that the equivariant cohomology $H_{G}^{*}(X)$ has been written in a normalized form with integrally defined generators. Let $n=\operatorname{edim} H^{*}(X)$ and take a $T^{n}$-principal bundle $\xi=\left\{T^{n} \rightarrow Y \rightarrow X\right\}$ as in Theorem 2.2. Then the $G$-action on $X$ can be lifted to a left $G$-bundle action on $\xi$ such that the $n$-tuple $c_{1}\left(\xi_{G}\right)=\left(X_{1}, \ldots, X_{n}\right) \in H^{2}\left(X_{G} ; \mathbf{Z}^{n}\right)$ is precisely the normalized generator set.

Lemma 5.1. Let the situation be as above. If $G$ acts c-effectively on $X$ then it acts on $Y$ with only finite isotropy groups.

Proof. Denote by $p: Y \rightarrow X$ the projection in the fiber bundle $\xi$, let $y \in Y$ and assume that the isotropy group $G_{v}$ has positive dimension, let $S \subset G_{y}^{0}$ be a maximal torus of the 1-connected component of $G_{y}$. It follows that $\operatorname{dim} S>0$. Let $x=p(y)$. Then, by the properties of a bundle action, the point $x$ is left fixed by $G_{y}$ and also by $S$. Then $S$ acts on the fiber $T_{x}=T^{n}$ by a homomorphism $\rho_{x}: S \rightarrow T^{n}$. Let $H_{G}^{*}(X)=P_{G} / I_{G}$ be a normalized presentation. After tensoring this with $R_{S}$ over $R_{G}$ we obtain a normalized form of $H_{S}^{*}(X)$ as $H_{S}^{*}(X) \cong P_{S} / I_{S}$ using the isomorphism $H_{S}^{*}(X) \cong H_{G}^{*}(X) \otimes_{R_{G}} R_{S}$. Let $F \subset X^{S}$ be the connected component with $x \in F$, and let $P \subset R_{S}$ be the corresponding associated prime ideal. Then $\operatorname{rk} P=$ rk $S$, because $S$ acts c-effectively. But by Theorem 2.2 we have $\operatorname{rk} \operatorname{Im} \rho_{x}=\operatorname{rk} P$. Thus it follows that $\operatorname{rk} \operatorname{Im} \rho_{x}=\operatorname{rk} S$; i.e., $\operatorname{ker} \rho_{x}$ is a finite subgroup of $S$. Now, $S$ acts on $T_{x}$ by left translations induced by the homomorphism $\rho_{x}$. Moreover, the point $y \in T_{x}$ is left fixed by $S$. Therefore we conclude $S \subset \operatorname{ker} \rho_{x}$, i.e., $S$ is a finite group. Because $S$ is connected we have $S=1$. Then it follows that $G_{y}^{0}$ is trivial, i.e., $G_{y}$ is a finite group, a contradiction.

Now, we recall the definition of an $F$-space. A space $X$ will be called an $F$-space if both pseudo-dual rational homotopy $\pi_{\psi}^{*}(X)$ and the rational cohomology $H^{*}(X)$ are finite-dimensional $\mathbf{Q}$-vector spaces. If $X$ is 1-connected this is equivalent to $\operatorname{dim}_{\mathbf{Q}} \pi_{*}(X) \otimes_{\mathbf{Z}} \mathbf{Q}<\infty$ and $\operatorname{dim}_{\mathbf{Q}} H^{*}(X)<\infty$. If, for example, $X=G / T, G$ a compact Lie group, $T \subset G$ a maximal torus, then $X$ is an $F$-space. If $X$ is a 
1-connected space define the homotopy Euler characteristic $\chi_{\pi}(X)$ of $X$ as

$$
\chi_{\pi}(X)=\sum_{j \geqslant 2}(-1)^{\operatorname{deg} \pi_{i}} \text { rk } \pi_{j}(X) \otimes_{\mathbf{z}} \mathbf{Q} .
$$

The following theorem is due to Halperin.

TheOREM (HALPeRIN [6]). Let $X$ be a 1-connected F-space. Then the following statements are equivalent:

(i) $\chi_{\pi}(X)=0$.

(ii) $\chi(X)>0, H^{*}(X ; \mathbf{Q})$ evenly graded.

(iii) $H^{*}(X) \cong \mathbf{Q}\left[x_{1}, \ldots, x_{n}\right] / I, \operatorname{deg} x_{i} \equiv O(2)$, where the ideal I is generated by a maximal regular series $\left\{f_{1}, \ldots, f_{n}\right\} \subset\left(x_{1}, \ldots, x_{n}\right)$; i.e., $H^{*}(X)$ is a local artinian positively graded complete intersection.

Thus it follows that the cohomology $H^{*}(X)$ of a 1-connected space $X$ of $q$-type which is also an $F$-space is a complete intersection. The converse is also true. The spaces considered in the above theorem are formal in the sense of rational homotopy theory, i.e., the minimal model $\mathfrak{M}(X)$ of such spaces is isomorphic as a differential graded algebra to the minimal model $\mathfrak{M}\left(H^{*}(X)\right)$ of the cohomology. The following lemma is a very easy consequence of the Koszul resolution:

Lemma 5.2. Let $A_{0}=\mathbf{Q}\left[x_{1}, \ldots, x_{n}\right] / I_{0}, I_{0}=\left(f_{1}, \ldots, f_{n}\right), \operatorname{deg} x_{i} \equiv O(2)$, where $\left\{f_{1}, \ldots, f_{n}\right\} \subset\left(x_{1}, \ldots, x_{n}\right)$ is a regular series of homogeneous elements. Then the minimal model $\mathfrak{M}\left(A_{0}\right)$ of $A_{0}$ is given by the graded commutative differential algebra:

$$
\begin{aligned}
\mathfrak{M}\left(A_{0}\right) & =\mathbf{Q}\left[\xi_{1}, \ldots, \xi_{n}\right] \otimes_{\mathbf{Q}} \Lambda\left(\beta_{1}, \ldots, \beta_{n}\right), \\
\operatorname{deg} \xi_{i} & =\operatorname{deg} x_{i}, \quad \operatorname{deg} \beta_{j}=\operatorname{deg} f_{j}-1,
\end{aligned}
$$

with the differential $d\left(\xi_{i}\right)=0$ and $d\left(\beta_{j}\right)=f_{j}\left(\xi_{1}, \ldots, \xi_{n}\right)$.

If $X$ is a 1-connected space it follows from rational homotopy theory that the dual rational homotopy group $\operatorname{Hom}_{\mathrm{z}}\left(\pi_{*}(X), \mathbf{Q}\right)$ is isomorphic as a graded vector space to the indecomposable quotient $\mathfrak{M}(X)_{+} / \mathfrak{M}(X)_{+}^{2}$. Let $X$ be a space of type $F$ and let $2 b_{1}-1, \ldots, 2 b_{q}-1,2 a_{1}, \ldots, 2 a_{r}$ be the positive integers occurring as the degrees of a homogeneous basis of $\operatorname{Hom}_{\mathbf{z}}\left(\pi_{*}(X), \mathbf{Q}\right)$. The integers $b_{1}, \ldots, b_{q}$; $a_{1}, \ldots, a_{r}$ will be called the $b$-exponents and the $a$-exponents of $X$.

EXAMPLE. If $G$ is a compact Lie group then it has only $b$-exponents which are at the same time the exponents of the corresponding Weyl group plus the exponents of an eventual central torus. So the compact Lie group $\mathrm{SU}(n)$ has the $b$-exponents $B=(2,3, \ldots, n)$, a torus $T$ of rank $r$ has $b$-exponents $B=(1,1, \ldots, 1)(r$-times).

EXAMPLE. If $X$ is a 1-connected $F$-space whose Q-cohomology is evenly graded, write $H^{*}(X)=\mathbf{Q}\left[x_{1}, \ldots, x_{n}\right] / I_{0}, I_{0}=\left(f_{1}, \ldots, f_{n}\right)$, with $\operatorname{deg} x_{i}=2 a_{i}, \operatorname{deg} f_{j}=2 b_{j}$. It follows from the above lemma that the $a$-exponents of $X$ are $A=\left(a_{1}, \ldots, a_{n}\right)$ and the $b$-exponents are $B=\left(b_{1}, \ldots, b_{n}\right)$. If in addition $X$ is a space of $q$-type, the $a$-exponents are given by $A=(1,1, \ldots, 1)$ and the $b$-exponents are given by $B=$ $\left(b_{1}, \ldots, b_{n}\right)$ with $b_{1}=2$, corresponding to the quadratic form $Q \in I_{0}$. 
Lemma 5.3. Let $X$ be a 1-connected F-space whose Q-cohomology is generated by elements of degree two. Take integrally defined generators $x_{1}, \ldots, x_{n} \in H^{2}(X)$ and consider the corresponding $T^{n}$-principal bundle $\xi=\left\{T^{n} \rightarrow Y \rightarrow X\right\}$. If $B=$ $\left(b_{1}, \ldots, b_{n}\right)$ are the b-exponents of $X$, then the b-exponents of $Y$ are also given by $B$. Moreover $Y$ is an $F$-space without a-exponents. (In fact it is rationally homotopy equivalent to a product of odd spheres $S^{2 b} j^{-1}, j=1, \ldots, n$.)

Proof. Write $H^{*}(X)=\mathbf{Q}\left[x_{1}, \ldots, x_{n}\right] /\left(f_{1}, \ldots, f_{n}\right)$. Then the minimal model $\mathfrak{M}(X)$ is given by

$$
\mathfrak{M}(X)=\mathbf{Q}\left[\xi_{1}, \ldots, \xi_{n}\right] \otimes_{\mathbf{Q}} \Lambda\left(\beta_{1}, \ldots, \beta_{n}\right),
$$

where the $\xi_{i}$ are cycles with homology classes $x_{i}$. Moreover we have $d\left(\beta_{j}\right)=$ $f_{j}\left(\xi_{1}, \ldots, \xi_{n}\right)$. The cohomology $H^{*}\left(T^{n}\right)$ of the fiber is a free graded commutative algebra whose generators $y_{1}, \ldots, y_{n} \in H^{1}\left(T^{n}\right)$ are completely transgressive in $\xi$ with transgression $\tau\left(y_{i}\right)=x_{i}$. Then the $n$-fold Hirsch extension

$$
ほ(Y)=\mathfrak{M}(X) \otimes_{\mathbf{Q}} \Lambda\left(\eta_{1}, \ldots, \eta_{n}\right),
$$

$\operatorname{deg} \eta_{i}=1, d\left(\eta_{i}\right)=\xi_{i}$, is a model of $Y$; see e.g. [17]. But $\&(Y)$ can be contracted to the minimal model

$$
\mathfrak{M}(Y)=\Lambda\left(\hat{\beta}_{1}, \ldots, \hat{\beta}_{n}\right)
$$

with $\operatorname{deg} \hat{\beta}_{j}=\operatorname{deg} f_{j}-1=2 b_{j}-1, d\left(\hat{\beta}_{j}\right)=0$.

Definition 5.1 (SEE [5]). Let $B=\left(b_{1}, \ldots, b_{q}\right), A=\left(a_{1}, \ldots, a_{r}\right)$ be two finite sequences of positive integers. We say that $(B, A)$ satisfies A.C. (arithmetic condition) if for every subsequence $A^{*}$ of $A$ of length $s, 1 \leqslant s \leqslant r$, there exist at least $s$ elements $b_{j}$ of $B$ of the form

$$
b_{j}=\sum_{a_{i} \in A^{*}} \gamma_{i j} a_{i},
$$

where the $\gamma_{i j}$ are nonnegative integers.

Our next result can then be formulated as follows.

TheOREM 5.3 (THEOREM 5). Let $X$ be a 1-connected $q$-space which is also an F-space and assume $X$ to be a locally finite $C W$-complex. Let $B^{X}=\left(b_{1}, \ldots, b_{n}\right)$ be the b-exponents of $X$. Let $G$ be a compact connected Lie group with b-exponents $B^{G}=$ $\left(c_{1}, \ldots, c_{r}\right)$. If $G$ acts c-effectively on $X$ in such a way that $X_{G}$ has the homotopy type of a $C W$-complex then the pair of sequences $B=B^{X}$ and $A=B^{G}$ satisfies $A$.C.

Proof. Take a $T^{n}$-principal bundle $\xi$ on $X$ as in Lemma 5.1 and lift the $G$-action to a left $G$-bundle action on $\xi$ such that the $n$-tuple $c_{1}\left(\xi_{G}\right)=\left(X_{1}, \ldots, X_{n}\right) \in$ $H^{2}\left(X_{G} ; \mathbf{Z}^{n}\right)$ is a normalized generator set of $H_{G}^{*}(X)$. After Lemma 5.1 the Lie group $G$ acts then on the total space $Y$ of $\xi$ with finite isotropy groups. By Lemma 5.3 the $b$-exponents of $Y$ are given by $B^{X}=\left(b_{1}, \ldots, b_{n}\right)$ and it has no $a$-exponents. Then it follows from Theorem 2 in [5] that the pair $B=B^{X}$ and $A=B^{G}$ satisfies A.C.

As a corollary we obtain

THEOREM 5.4 (THEOREM 6). Let $X$ be a 1-connected space of q-type which is also an $F$-space and assume $X$ to be a locally finite $C W$-complex. Let $G$ be a compact connected semisimple Lie group which acts c-effectively on $X$ in such a way that $X_{G}$ has 
the homotopy type of a $C W$-complex. If $T \subset G$ is a maximal torus then the Poincarépolynomial $P(G / T ; t)$ of $G / T$ divides the Poincare polynomial $P(X ; t)$ of $X$ such that the quotient $P(X ; t) / P(G / T ; t)$ is a polynomial with nonnegative integral coefficients.

Proof. Because $H^{*}(X)$ is a complete intersection, the Poincaré-polynomial of $X$ is given by

$$
P(X ; t)=\prod_{j=1}^{n}\left(1+t^{2}+\cdots+t^{2\left(b_{i}-1\right)}\right),
$$

where the $b_{j}, j=1, \ldots, n$, are the $b$-exponents of $X$. Moreover, the Poincaré-polynomial of $G / T$ is given by

$$
P(G / T ; t)=\prod_{i=1}^{r}\left(1+t^{2}+\cdots+t^{2\left(c_{i}-1\right)}\right),
$$

where the $c_{i}, i=1, \ldots, r$, are the $b$-exponents of $G$. By Theorem 5.3 the pair $B^{X}=\left(b_{1}, \ldots, b_{n}\right), B^{G}=\left(c_{1}, \ldots, c_{k}\right)$ satisfies A.C. Then the statement follows from Proposition 3.1 in [5].

-THEOREM 5.5. Let $X$ be a space of $q$-type. If a compact connected semisimple Lie group $G$ acts c-effectively on $X$, then there exists a surjective $R_{G}$-algebra homomorphism $\varphi: H_{G}^{*}(X) \rightarrow H_{G}^{*}(G / T)$, where $H_{G}^{*}(G / T) \cong R_{T}$ is the equivariant cohomology of the natural $G$-action on $G / T$ by left translations. In particular, there exists $a$ surjective homomorphism of graded Q-algebras $\varphi$ : $H^{*}(X) \rightarrow H^{*}(G / T)$.

ProOf (SEE ALSO [10]). We consider the following commutative diagram.

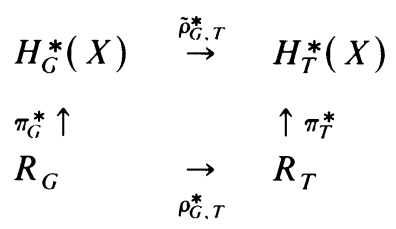

Here we have the isomorphism $H_{T}^{*}(X) \cong H_{G}^{*}(X) \otimes_{R_{G}} R_{T}$, as follows from Theorem 2.2. We observe that the choice of a fixed point $q \in X^{T}$ induces a section $s$ in the fibration $X_{T} \rightarrow B_{T}$ as follows: If $b \in B_{T}$ choose an element $e \in E_{T}$ which projects to $b$. Then put $s(b)=[e, q] \in E_{T} \times_{T} X$. It follows $\pi_{T} \circ s=\mathrm{id}_{B_{T}}$; i.e., $s$ is a section. In particular, we have $s \circ \pi_{T}^{*}=\mathrm{id}_{R_{T}}$. Define $\varphi$ as the composition $\varphi=s^{*} \circ \tilde{\rho}^{*}$. We then have the commutative triangle

$$
\begin{array}{llll}
H_{G}^{*}(X) & \stackrel{\varphi}{\rightarrow} & R_{T} \\
\pi_{G}^{*} \nwarrow & & \\
& & R_{G} &
\end{array}
$$

as can be shown by a simple diagram chasing argument. In other terms $\varphi$ is a graded $R_{G}$-algebra homomorphism. We show that $\varphi$ is indeed an epimorphism. Let the equivariant cohomology $H_{G}^{*}(X)$ be given in the normalized form $H_{G}^{*}(X)=P_{G} / I$, and let $J \subset P_{T}$ be the ideal generated by $I$ in the ring $P_{T}$ using the canonical injection $P_{G} \subset P_{T}$. Let $F$ be the fixed space component of $T$ containing $q$. Let 
$P=\left(X_{1}-\omega_{1}, \ldots, X_{n}-\omega_{n}\right) \subset P_{T}$ be the corresponding prime ideal. Then $s^{*}$ : $H_{T}^{*}(X) \rightarrow R_{T}$ is given by $s^{*}\left(\bar{X}_{i}\right)=\omega_{i}$. Here $\bar{X}_{i} \in H_{T}^{*}(X)$ is the projection of $X_{i} \in P_{T}$ down to $H_{T}^{*}(X)$. It follows that $\varphi$ is given by $\varphi\left(\bar{X}_{i}\right)=\omega_{i}, i=1, \ldots, n$. By Lemma $3.3 \operatorname{rk}\left(\omega_{1}, \ldots, \omega_{n}\right)=\operatorname{rk} T$, so $\varphi$ must be an epimorphism. Tensoring the homomorphism $\varphi$ over $R_{G}$ with $\mathbf{Q}$ gives an epimorphism $\bar{\varphi}: H^{*}(X) \rightarrow H^{*}(G / T)$.

As a corollary we obtain

THEOREM 5.6. Let $X$ be a space of q-type. If a compact connected semisimple Lie group $G$ acts c-effectively on $X$ then $\operatorname{cd}_{\mathbf{Q}} G / T \leqslant \operatorname{cd}_{\mathbf{Q}} X$.

The proof is clear and shall be omitted.

THEOREM 5.7. Let $X$ be a space of q-type. If a compact connected semisimple Lie group $G$ acts c-effectively on $X$ then the fixed space $X^{G}$ is always empty.

Proof. If $X^{G}$ is nonempty, pick a connected component $X_{0}^{G}$ of $X^{G}$. Then $X_{0}^{G}$ is contained in a connected component $F$ of $X^{T}$ where $T$ is a maximal torus of $G$. Now $x \in X_{0}^{G}$ is fixed by $N T$, the normalizer of $T$ in $G$. By continuity reasons it follows that $[F] \in \pi_{0} X^{T}$ is fixed by $W G=N T / T$ which is not possible by Theorem 5.1 .

THEOREM 5.8. Under the hypotheses of Theorem 5.7 the isotropy group $G_{x} \subset G$ of a point $x \in X$ is always the extension of a finite group $K$ with a torus.

This follows immediately from Theorem 5.7.

We can sharpen the result of Theorem 5.7 as follows.

THEOREM 5.9. Under the hypotheses of Theorem 5.7 the isotropy groups $G_{x} \subset G$ of points $x \in X^{T}$ are always maximal tori; in particular, they are connected.

Proof. Let $x \in X^{T}$ and $G_{x}$ be its isotropy group. Then the 1-connected component $G_{x}^{0}$ is equal to $T$. By continuity reasons the finite group $K=G_{x} / G_{x}^{0}$ acts on the connected component $F$ of $X^{T}$ containing $x$. But $G_{x}^{0}=T$ is normal in $G_{x}$, so $K$ must be a subgroup of $W G$. But then, by Theorem 5.1 again, $K$ must be the trivial subgroup.

Let $X^{(T)} \subset X$ be the set of all points in $X$ whose isotropy group $G_{x}$ has maximal rank. Then $X^{(T)}$ is a closed $G$-invariant subset of $X$.

THEOREM 5.10. Under the hypotheses of Theorem 5.7 the set $X^{(T)}$ is a disjoint union of spaces $F_{j} \times G / T$, where $F_{j}$ is a connected component of $X^{T}$ and $j$ runs through $W G \backslash \pi_{0} X^{T}$.

Proof. This follows from the fact that the action of a connected semisimple Lie group $G$ with only connected isotropy groups of maximal rank on a connected space $Y$ is completely determined by the $W G$-action on $Y^{T}$ (see $[\mathbf{8}]$ ).

We come now to the application of our results to $G$-actions on homogeneous spaces $K / T_{K}$, where $K$ is a semisimple compact Lie group and $T_{K} \subset K$ its maximal torus. 
THEOREM 5.11. Let $G$ be a semisimple compact Lie group, and let $X$ be a compact connected closed oriented manifold which is a space of q-type. Moreover assume $\chi(X)=|W G|$. If $G$ acts effectively on $X$, then $X=G / T$ and $G$ acts on $X$ by left translations.

Proof. As in the proof of Theorem 3.2 it follows that $G$ acts c-effectively. By Theorem 5.5 there exists a surjective graded ring homomorphism $\varphi: H^{*}(X) \rightarrow$ $H^{*}(G / T)$ which in this case must be an isomorphism. So it follows that $\operatorname{cd}_{\mathrm{Q}} X=$ $\operatorname{cd}_{\mathbf{Q}} G / T$. But $X$ and $G / T$ are compact closed connected oriented manifolds. Thus it follows that $\operatorname{dim} X=\operatorname{dim} G / T$. By Theorem 5.9 there exists an orbit $G(x)=G / T$ in $X$. So we have $X=G / T$ and $G$ acts on $X$ by left translations up to conjugation.

THEOREM 5.12 (THEOREM 7). Let $X$ be a space of q-type. If a compact connected semisimple Lie group $G$ acts c-effectively on $X$ then $\operatorname{dim} G \leqslant \operatorname{cd}_{\mathbf{Q}} X+\operatorname{edim} H^{*}(X)$.

Proof. If a compact semisimple Lie group $G$ acts c-effectively on $X$, then, by Theorem $5.6 \operatorname{cd}_{\mathbf{Q}} G / T \leqslant \operatorname{cd}_{\mathbf{Q}} X$. This gives $\operatorname{dim} G / T \leqslant \operatorname{cd}_{\mathbf{Q}} X$. By Theorem 3.1 rk $G \leqslant \operatorname{edim} H^{*}(X)$. So $\operatorname{dim} G \leqslant \operatorname{cd} Q+\operatorname{edim} H^{*}(X)$.

THEOREM 5.13 (THEOREM 8). If $X$ is a compact connected closed oriented manifold of q-type, then $N_{t}^{\mathrm{ss}}(X) \leqslant \operatorname{dim} X+\operatorname{edim} H^{*}(X)$.

PROOF. In the proof of Theorem 3.2 it has been shown that an effective action of $G$ on $X$ is also c-effective. (The argument used is also valid for an arbitrary compact Lie group.) Then the result follows from Theorem 5.12.

THEOREM 5.14 (THEOREM 9). Let $G$ be a compact semisimple Lie group, $T \subset G a$ maximal torus, and let $X=G / T$. Then $N_{t}(X)=N_{t}^{\mathrm{ss}}(X)=\operatorname{dim} G$. Moreover, if a compact Lie group $K$ with $\operatorname{dim} K=\operatorname{dim} G$ acts effectively on $X$, then $X$ is locally isomorphic to $G$ and $K$ acts on $X$ by left translations up to conjugation.

Proof. By Theorem 5.13 we have $N_{t}^{\text {ss }}(X) \leqslant \operatorname{dim} G$; because $G$ acts effectively on $X$ by left translations, we get $N_{t}^{\text {ss }}(X)=\operatorname{dim} G$. Suppose a compact Lie group $K=S \times Z, S$ semisimple, $Z$ a central torus, acts effectively on $X$. Then it acts c-effectively and we have

$$
\begin{aligned}
& \operatorname{dim} S-\operatorname{rk} S \leqslant \operatorname{dim} G-\operatorname{rk} G \quad \text { (Theorem 5.6), } \\
& \text { rk } S+\text { rk } Z \leqslant \text { rk } G \quad \text { (Theorem 3.1). }
\end{aligned}
$$

But $\operatorname{dim} K=\operatorname{dim} S+$ rk $Z$, so the addition of both inequalities gives $\operatorname{dim} K \leqslant \operatorname{dim} G$. So $N_{t}(X)=N_{t}^{\text {ss }}(X)=\operatorname{dim} G$. Now assume $\operatorname{dim} K=\operatorname{dim} G$. If $\operatorname{dim} S-\mathrm{rk} S<$ $\operatorname{dim} G-\operatorname{rk} G$, it follows by the second inequality that $\operatorname{dim} K=\operatorname{dim} S+\operatorname{rk} Z<$ $\operatorname{dim} G$, a contradiction. So we have $\operatorname{dim} S-\operatorname{rk} S=\operatorname{dim} G-\operatorname{rk} G$; i.e., $\operatorname{dim} S / T_{S}=$ $\operatorname{dim} G / T$, where $T_{S} \subset S$ is a maximal torus of $S$. Let $\varphi: S \times X \rightarrow X$ be the induced action. By Theorem 5.9 the space $X$ contains an orbit $S(X)=S / T_{S}$. It follows that $X=G / T=S / T_{S}$. But then we have $\operatorname{rk} G=\operatorname{rkS}$ and by Theorem 3.1 it follows that rk $Z=0$; i.e., $K=S$ is semisimple. Thus $K$ acts on $X$ by left translations. It is an easy exercise to show that $G / T=S / T_{S}$ implies that $G$ is locally isomorphic to $S$. (Hint: Use the lifting theorem of Hattori and Yoshida and show that the lifted $S$-action on $G$ is almost free.) 
6. Lie groups which cannot act on a given manifold. In this section we wish to exploit the divisibility condition of Theorem 5.2 a bit more. This theorem gives very strong restrictions for the possible semisimple Lie groups which can act on a space $X$ of $q$-type. The orders of Weyl groups of simple Lie groups are given in the following table

$\begin{array}{lll}\operatorname{SU}(n) & (n+1) ! & n \geqslant 2 \\ \operatorname{Spin}(2 n+1) & 2^{n} n ! & n \geqslant 2 \\ \operatorname{Sp}(n) & 2^{n} n ! & n \geqslant 2 \\ \operatorname{Spin}(2 n) & 2^{n-1} n ! & n \geqslant 3 \\ G_{2} & 2^{2} \cdot 3 & \\ F_{4} & 2^{7} \cdot 3^{2} & \\ E_{6} & 2^{7} \cdot 3^{4} \cdot 5 & \\ E_{7} & 2^{10} \cdot 3^{4} \cdot 5 \cdot 7 & \\ E_{8} & 2^{14} \cdot 3^{5} \cdot 5^{2} \cdot 7 & \end{array}$

Let $X$ be a closed compact 1-connected manifold of $q$-type such that $H^{*}(X)$ is a complete intersection. If we write

$$
H^{*}(X)=\mathbf{Q}\left[x_{1}, \ldots, x_{n}\right] /\left(f_{1}, \ldots, f_{n}\right)
$$

with $\operatorname{deg} x_{i}=2, \operatorname{deg} f_{j}=2 b_{j}$, then

$$
\chi(X)=\prod_{j=1}^{n} b_{j}, \quad \mathrm{~cd}_{\mathbf{Q}} X=2\left(\sum_{j=1}^{n} b_{j}-n\right)
$$

as follows from the formulas in [5, 2]. Due to the existence of a quadratic form $Q$ in the defining ideal $I_{0}=\left(f_{1}, \ldots, f_{n}\right)$ there must be at least one $f_{j}$, say $f_{1}$, with $\operatorname{deg} f_{1}=\operatorname{deg} Q=4$. So it follows that $\chi(X) \equiv O(2)$. This implies that the groups $G=\operatorname{Spin}(3)$ or $G=\mathrm{SO}(3)$ can never be excluded as possible acting groups by Theorem 5.2. Let us give an example. Take $c=500,000,000,000=5 \cdot 10^{11}$. (In marks this corresponds to the contemporary German national debt, December 1984.) Then $c$ has the prime number decomposition $c=2^{11} \cdot 5^{12}$. If $X$ is a manifold as above ( $=q$-manifold) with $\chi(X)=c$ and a compact semisimple Lie group $G$ acts effectively on $X$, then $|W G|$ divides $2^{11} 5^{12}$. By the Table it is quite easy to see that then $G$ must be locally isomorphic to $\operatorname{Spin}(3)^{i}, 1 \leqslant i \leqslant 11$. The highest possible dimension a $q$-manifold $X$ with $\chi(X)=c$ can have is given by $\operatorname{dim} X=c$. This is the case when $H^{*}(X)$ has embedding dimension equal to 2; i.e.,

$$
H^{*}(X)=\mathbf{Q}[x, y] /\left(f_{1}, f_{2}\right),
$$

$\operatorname{deg} f_{1}=4, \operatorname{deg} f_{2}=c$. So, in this case the rank of the acting group is restricted by rk $G \leqslant 2$. Then the highest dimensional compact Lie group $G$ which cannot be excluded is locally isomorphic to $\operatorname{Spin}(3) \times \operatorname{Spin}(3)$; i.e., $N_{t}(X) \leqslant 6$. The opposite case, i.e., when $X$ has the lowest possible dimension, is represented by a cohomology ring of the form

$$
H^{*}(X) \cong \mathbf{Q}\left[x_{1}, \ldots, x_{23}\right] /\left(f_{1}, \ldots, f_{11}, g_{1}, \ldots, g_{12}\right),
$$


where $\operatorname{deg} f_{j}=4, \operatorname{deg} g_{k}=10$. The corresponding cohomology dimension is then $\operatorname{cd}_{\mathbf{Q}} X=\operatorname{dim} X=118$. The rank of a torus $T$ which acts effectively on $X$ is restricted by rk $T \leqslant 23$. The highest dimensional compact Lie group $G$ which can possibly act on $X$ is locally isomorphic to $\operatorname{Spin}(3)^{11} \times T^{12}$; i.e., $N_{t}(X) \leqslant 45$. Thus we see that although the very high Euler number $c$ there is not much room for actions. (Perhaps one should wait until the national debt has reached the order of the next possible Weyl group!) The following estimation of the semisimple symmetry degree of $q$-manifolds is surely much stronger then the upper bound given in Theorems 5.12 and 5.13. Nevertheless it is a simple consequence of Theorem 5.2. We define a number theoretical function $\Phi: \mathbf{N} \rightarrow \mathbf{N}$ as

$$
\Phi(n)=\max \{\operatorname{dim} G ; G \text { compact semisimple, }|W G| / n\} .
$$

Then we have

THEOREM 6.1 (THEOREM 10). If $X$ is a compact connected closed oriented manifold of q-type, then $N_{t}^{\mathrm{ss}}(X) \leqslant \Phi(\chi(X))$.

If $n$ is an even positive integer such that $n \not \equiv O(4)$ and $n \not \equiv O(6)$ then $\Phi(n)=$ $\operatorname{dim} \operatorname{Spin}(3)=3$. Thus we obtain

THEOREM 6.2. If $X$ is a compact connected closed oriented manifold of q-type with $\chi(X) \not \equiv O(4)$ and $\chi(X) \not \equiv O(6)$, then $N_{t}^{\text {ss }}(X) \leqslant 3$. If a compact connected semisimple Lie group $G$ acts effectively on $X$ then $G$ is locally isomorphic to $\operatorname{Spin}(3)$.

It would be interesting to know whether a compact Lie group $G$ which cannot be excluded by Theorem 5.2 does indeed act on a space of $q$-type with the prescribed Euler number. This would include constructions of a wide range of $G$-actions on such spaces, a plan which goes beyond the scope of this paper. Nonetheless we prove the following preliminary result.

THEOREM 6.3. Let $c=2 m$ be an even positive integer. Then there exists a finite $C W$-complex $X$ of $q$-type, $\chi(X)=c$, with a cellular $\operatorname{Spin}(3)$-action which is effective.

Proof. Consider the graded Q-algebra

$$
A_{0}=\mathbf{Q}[x, y] /\left(f_{1}, f_{2}\right), \quad \operatorname{deg} x=\operatorname{deg} y=2,
$$

$f_{1}=x^{2}+y^{2}, f_{2}=x^{a} y^{b}, a+b=m, a>0, b>0$. Then $A_{0}$ is a complete intersection algebra with $\operatorname{dim}_{\mathrm{Q}} A_{0}=2(a+b)=c$. We construct a finite CW-complex $X$, $H^{*}(X) \cong A_{0}$, with a nontrivial Spin(3)-action on it. We have

$$
H^{*}\left(B_{\mathrm{Spin}(3)}\right) \cong \mathbf{Q}[s] \subset \mathbf{Q}[t] \cong H^{*}\left(B_{T^{1}}\right)
$$

where the inclusion is given by $s \rightarrow t^{2}$. Now, we construct a graded flat $\mathrm{Q}[s]$-algebra $A$ with $A \otimes_{\mathrm{Q}[s]} \mathbf{Q}=A_{0}$. Later this will be the equivariant cohomology ring $H_{\text {Spin(3) }}^{*}(X)$. Write $G=\operatorname{Spin}(3)$, take $P_{G}=R_{G}[x, y]$ and consider the ideal $I \subset P_{G}$ generated by the homogeneous elements $F_{1}=x^{2}+y^{2}-s$ and $F_{2}=F$ which is 
given as follows

$$
\begin{array}{ll}
F=\prod_{i=1}^{\alpha}\left(x^{2}-\omega_{i}^{2} s\right) \cdot \prod_{j=1}^{\beta}\left(y^{2}-\lambda_{j}^{2} s\right), & \text { if } a=2 \alpha, b=2 \beta, \\
F=x \cdot \prod_{i=1}^{\alpha}\left(x^{2}-\omega_{i}^{2} s\right) \cdot \prod_{j=1}^{\beta}\left(y^{2}-\lambda_{j}^{2} s\right), & \text { if } a=2 \alpha+1, b=2 \beta, \\
F=\prod_{i=1}^{\alpha}\left(x^{2}-\omega_{i}^{2} s\right) \cdot y \cdot \prod_{j=1}^{\beta}\left(y^{2}-\lambda_{j}^{2} s\right), & \text { if } a=2 \alpha, b=2 \beta+1, \\
F=x \cdot \prod_{i=1}^{\alpha}\left(x^{2}-\omega_{i}^{2} s\right) \cdot y \cdot \prod_{j=1}^{\beta}\left(y^{2}-\lambda_{j}^{2} s\right), & \text { if } a=2 \alpha+1, b=2 \beta+1 .
\end{array}
$$

Here the $\omega_{i}, \lambda_{j}$ are rational numbers such that $\sqrt{1-\omega_{i}^{2}}$ and $\sqrt{1-\lambda_{j}^{2}}$ are again rational and such that $\omega_{i}^{2}+\lambda_{j}^{2} \neq 1$ for all pairs $(i, j)$. (There are enough pythagorean numbers.) If $A=P_{G} / I$, then clearly, $A \otimes_{\mathrm{Q}[s]} \mathbf{Q}=A_{0}$. We consider the ideals $I_{0}$, $J_{0}, I_{i}, i=1, \ldots, \alpha, J_{j}, j=1, \ldots, \beta$, with

$$
\begin{aligned}
& I_{0}=\left(x^{2}+y^{2}-s, x\right), \quad J_{0}=\left(x^{2}+y^{2}-s, y\right), \\
& I_{i}=\left(x^{2}+y^{2}-s, x^{2}-\omega_{i}^{2} s\right), \quad J_{j}=\left(x^{2}+y^{2}-s, y^{2}-\lambda_{j}^{2} s\right) .
\end{aligned}
$$

We then have the primary decompositions

$$
\begin{aligned}
& I=\bigcap_{i=1}^{\alpha} I_{i} \cap \bigcap_{j=1}^{\beta} J_{j}, \\
& I=I_{0} \cap \bigcap_{i=1}^{\alpha} I_{i} \cap \bigcap_{j=1}^{\beta} J_{j}, \\
& I=\bigcap_{i=1}^{\alpha} I_{i} \cap J_{0} \cap \bigcap_{j=1}^{\beta} J_{j}, \\
& I=I_{0} \cap \bigcap_{i=1}^{\alpha} I_{i} \cap J_{0} \cap \bigcap_{j=1}^{\beta} J_{j},
\end{aligned}
$$

depending on the four possibilities above. We have then natural exact sequences of the following kind (here we write it down only for the first case)

$$
0 \rightarrow P_{G} / I \rightarrow \prod_{i=1}^{\alpha} P_{G} / I_{i} \oplus \prod_{j=1}^{\beta} P_{G} / J_{j} \rightarrow C \rightarrow 0
$$

The cokernel $C$ is a finitely generated $R_{G}$-torsion module; thus it follows that $C$ is a finite dimensional graded $\mathbf{Q}$-vector space. This follows from the fact that $R_{G}=\mathbf{Q}[s]$ is a principal ideal domain. We consider the different summands in the middle term. These are the rings $P_{G} / I_{0}, P_{G} / I_{i}, P_{G} / J_{0}$ and $P_{G} / J_{j}$. The ring $P_{G} / I_{0}$ is isomorphic to 
the ring $R_{G}[y] /\left(y^{2}-s\right)$. But this is indeed the equivariant cohomology of the natural Spin(3)-action on the 2-sphere; i.e.,

$$
P_{G} / I_{0} \cong H_{G}^{*}\left(S^{2}\right) \text {. }
$$

The same holds for the ring $P_{G} / J_{0}$. We claim that $P_{G} / I_{i}$ is isomorphic to the equivariant cohomology $H_{G}^{*}\left(S^{2} \times S^{2}\right)$, where $G=\operatorname{Spin}(3)$ acts on $S^{2} \times S^{2}$ by the natural diagonal action. It is easy to see that we have the following identities:

$$
\begin{aligned}
P_{G} / I_{i} & =R_{G}[x, y] /\left(x^{2}+y^{2}-s, x^{2}-\omega_{i}^{2} s\right) \\
& =R_{G}[x, y] /\left(x^{2}-\omega_{i}^{2} s, y^{2}-\left(1-\omega_{i}^{2}\right) s\right) \\
& =R_{G}[x, y] /\left(x^{2}-s, y^{2}-s\right),
\end{aligned}
$$

where the last isomorphism is given by $x \rightarrow \omega_{i} x, y \rightarrow \sqrt{1-\omega_{i}^{2} y}$. But the last ring is indeed isomorphic to $H_{G}^{*}\left(S^{2} \times S^{2}\right)$. Then we begin the construction of the $\operatorname{Spin}(3)-$ complex as follows: We take $\alpha+\beta$ copies of $S^{2} \times S^{2}$ with diagonal Spin(3)-action and add one or two 2-spheres with Spin(3)-action. The disjoint union of these $G$-spaces will be called $Y$. Then $H_{G}^{*}(Y)$ is the middle term in the above exact sequence. Now, $Y_{G}=E_{G} \times{ }_{G} Y$ is a formal space in the sense of rational homotopy theory. Along the same lines as in the proof of Theorem 2.1 in [16] we can adjoin free $G$-cells $G \times e^{N}$ to $Y$ following the pattern of the $R_{G}$-module $C$. The resulting space $X$ is a $G$-space with $H_{G}^{*}(X) \cong P_{G} / I=A$. Thus it follows $H^{*}(X) \cong A_{0}$. In particular, the sequence

$$
0 \rightarrow H_{G}^{*}(X) \rightarrow H_{G}^{*}(Y) \rightarrow H_{G}^{*+1}(X, Y) \rightarrow 0
$$

is exact and coincides with $(*)$. We remark that the fixed point set $X^{S^{1}}$ of the induced $S^{1}$-action is equal to $Y^{S^{1}}$ and is therefore a union of isolated points.

\section{REFERENCES}

1. A. Borel, Topics in the homologv theory of fiber bundles, Lectures Notes in Math., vol. 36, Springer-Verlag, Berlin and New York, 1967.

2. T. tom Dieck, Lokalisierung äquivarianter Kohomologietheorien, Math. Zeit. 121 (1971) 253-262.

3. E. B. Dynkin, Semisimple subalgebras of semisimple Lie algebras, Amer. Math. Soc. Transl. (2) 6 (1957), 111-244.

4. , The maximal subgroups of the classical groups, Amer. Math. Soc. Transl. (2) 6 (1957), $245-378$.

5. J. B. Friedländer and S. Halperin, An arithmetic characterization of the rational homotopy groups of certain spaces, Invent. Math. 53 (1979), 117-133.

6. S. Halperin, Finiteness in the minimal models of Sullivan, Trans. Amer. Math. Soc. 230 (1977), $173-199$.

7. A. Hattori and T. Yoshida, Lifting compact group actions in fiber bundles, Japan J. Math. 2 (1976), 13-25.

8. V. Hauschild, Compact Lie group actions with isotropy subgroups of maximal rank, Manuscripta Math. 34 (1981), 355-379.

9. __ Deformationen graduierter artinscher Algebrèn in der Kohomologietheorie von Transformationsgruppen, Habilitationsschrift, Konstanz, 1984.

10. Actions of compact Lie groups on homogeneous spaces, Math. Z. 189 (1985), 475-486.

11. K. Hokama and S. Kono, On the fixed point set of $S^{1}$-actions on the complex flag manifolds, Math. J. Okayama Univ. 20 (1978), 1-16. 
12. W. Y. Hsiang, Cohomology theory of topological transformation groups, Ergeb. Math. Grenzgeb. Band 85, Springer-Verlag Berlin and New York, 1975.

13. T. Y. Lam, The algebraic theory of quadratic forms, Benjamin/Cummings, Reading, Mass., 1973.

14. H. Matsumura, Commutative algebra, Benjamin, New York, 1970.

15. V. Puppe, On a conjecture of Bredon, Manuscripta Math. 12 (1974), 11-16.

16. 119-136.

17. P. Deligne, $\mathrm{Ph}$. Griffiths, and J. Morgan, Real homotopy of Kähler manifolds, Invent. Math. 29 (1979), 245-274.

Fakultät fÜr Mathematik, Universität Konstanz, Universitätsstrasse 1, D - 7750 Konstanz. Fi:DERAL. REPUBLIC OF GERMANY

Sonderforschungsbereich “Geometrie und Analysis”, Bunsenstrasse 3 - 5, D - 3400 GOTtingen, Federai. Repubicic of Germany 